\title{
EL FUTURO DE LAS HUMANIDADES Y DE LAS CIENCIAS SOCIALES: EL IMPERATIVO DE UNA REFORMA
}

\author{
Alfonso De Toro $\left(^{*}\right)$
}

\section{El estado de la cuestión: la situación precaria de las Humanidades y de las ciencias sociales}

¿De qué trata el tema de mi conferencia hoy?

Trata del éxito o del fracaso de las sociedades en el mundo actual.

Trata del éxito o del fracaso de la economía, de las ciencias y de la técnica.

Trata del éxito o del fracaso del bienestar de las sociedades actuales.

Trata del éxito o del fracaso de impartir una educación y darle bienestar económico y cultural a toda una sociedad, independientemente del origen y del nombre de las personas.

Este bienestar está basado en el éxito o en el fracaso de los sistemas de educación primaria, secundaria y superior; en el éxito o en el fracaso de la configuración de una sociedad de conocimiento, que sea capaz de competir mundialmente, esto es, de ser dueña de sus productos, dueña de su ciencia, dueña de su saber y conocimiento y no de mantener la dependencia del extranjero en forma perenne, a través de la importación tecnología ${ }^{1}$.

Este debate se concentra y gira en torno a la Economía y a las Ciencias Exactas

${ }^{*}$ ) Centro de Investigación Iberoamericana, Universidad de Leipzig.

Conferencia magistral dictada en la Universidad de Talca, en el XXVI aniversario de su creación, 26 de octubre de 2007.

1 Así se manifestó la Directora de Postgrado de la Universidad de Chile, Dra. Rosa Devés en El Mercurio del 27 de octubre 2007 con motivo de la manifestación pública de científicos chilenos a raíz del corte de presupuesto para la investigación. 
(naturales y de ingeniería); las Humanidades y las Ciencias Sociales ${ }^{2}$ quedan más o menos marginadas en la discusión y presencia pública, y al parecer fuera de toda función pasando, aparentemente, a ser innecesarias. Por ello, mi tema será hacer una breve radiografía del estado de ciertos aspectos de la discusión y de nuestra sociedad y educación, y demostrar la imprescindible necesidad de las Humanidades y de las Ciencias Sociales en la acción de una sociedad y universidad. Asimismo, dentro de este contexto, propondré una cartografía para una alternativa de reforma de estructuras universitarias dentro de las Humanidades y las Ciencias Sociales.

Así como en el mundo actual fenómenos de tipo económico, científico, tecnológico y ecológico se tratan en un contexto global, partiendo de estándares similares, de igual forma se debe proceder imprescindiblemente con la educación tanto primaria como secundaria y superior. El sistema de evaluación Programme for International Student Assessment (PISA) para la educación escolar o la Organisation for Economic Co-operation and Development (OECD) y los permanentes rankings universitarios -el último de la Universidad de Shangai-, son claras concretizaciones del trato global que se le da a la educación hoy en día.

Por ello, si hablamos de la reforma educacional, escolar o universitaria y del futuro de las Humanidades y de las Ciencias Sociales lo debemos hacer en un contexto internacional, considerando estándares internacionales. La discusión sobre los estándares de educación se está dando en este momento en todas partes del mundo.

El primer paso es realizar un diagnóstico del estado de la educación escolar y superior dentro de parámetros internacionales y preguntarnos para qué sirve la educación que estamos impartiendo.

En el caso de Latinoamérica, y particularmente de Chile, el Rector de la Universidad Católica del Norte, Misael Camus, dictó en Múnich el 5 de octubre de 2006 en el encuentro del Consejo de Rectores de las Universidades Chilenas (CRUCH) y de la Hochschulrektorenkonferenz (HRK, conferencia de rectores universitarios) una conferencia con el título Current Trends in Highier Education in Chile donde dejaba en claro que Latinoamérica, ni aun Brasil o México, prácticamente no tiene ninguna representación en el ámbito internacional, ya sea en la producción de profesionales o en la producción de altas y nuevas tecnologías ni, por ende, en la producción de patentes de inventos, ni en la economía, ni en las ciencias. Hizo, además, una comparación de las universidades latinoamericanas y chilenas, constatando que la centralización de la producción en Santiago, por ejemplo, representa uno de los tantos problemas por resolver. Este análisis, conectado con aquellos de PISA -donde la educación alemana, por ejemplo, sale bastante mal evaluada-, o con el último reportaje de la $O E C D$-en el cual Alemania cae del puesto 10 al 22 y en el que se critica que haya muy pocos bachilleres y absolventes, y que las cifras de estudiantes

2 A pesar de que los campos de investigación y de docencia de las Ciencias de las Humanidades y de las Ciencias Sociales son distintos no los diferencio en mi argumentación ya que ambos sectores -que se resumen en alemán como las "Geisteswissenschaften"- tienen similares problemas de legitimación y de reformulación. Naturalmente que aquellos campos de trabajo empírico en las Ciencias Sociales y en las Ciencias Políticas, como encuestas y estadísticas, no están afectados por los problemas a tratar en las siguientes reflexiones. 
que no terminan los estudios sean muy altas-, y así con el último ranking de la Universidad de Shangai-aunque, especialmente en el caso de este ranking, los criterios usados para evaluar las universidades sean criticables-, habla un lenguaje clarísimo.

Dentro de las 100 mejores universidades en Norte y Sudamérica, según este ranking, se encuentran solamente dos universidades latinoamericanas, la UBA y la UNAM, entre el lugar 77 y 88, y mundialmente se encuentran entre el lugar 151 y 202.

En el ranking tan sólo aparecen dos universidades chilenas, la Universidad Católica y la Universidad de Chile, y se encuentran en el ámbito mundial entre el lugar 403 y 500, y en América entre el lugar 165 y el 197. Con lo que respecta a las universidades alemanas, la Maximiliam Universität y la Technische Universität München ocupan los lugares 53 y 56, entre los 100 primeros del mundo (en Europa el 11 y el 14); la Universidad de Heidelberg ocupa el lugar 65 en el mundo (el 17 en Europa); la de Gottinga, el 86 en el mundo (el 29 en Europa); la de Friburgo, el 94 en el mundo (el 31 en Europa), y la de Bonn, el 99 en el mundo (el 33 en Europa). Y estas universidades alemanas han sido en 2007 definidas por la Deutsche Forschungsgemeinschaft (German Research Counsil) como de elite, por ello, parece ser que los criterios de la Universidad de Shangai son bastante certeros.

Los resultados de las estadísticas mencionadas nos debe dar que pensar, especialmente en el caso de Chile con su ambicioso plan de pasar a ser un país con competencias fundamentales.

En los años ochenta el tema del estado de la universidad y de las Humanidades y de las Ciencias Sociales se discutió particularmente en Alemania en un gran número de suplementos de grandes periódicos y revistas especializadas, y se trató de igual forma en proyectos científicos y publicaciones científicas (vid., por ejemplo, Frühwald et alii 1991/21996; Gayer 1997). Hoy se vuelve a presentar la discusión a la vez en forma similar y diferente.

Para enfrentar al tema en cuestión quisiera formular algunas premisas que serán la base de mi exposición.

\section{Competencia en el ámbito internacional}

Si un país quiere tener éxito, esto es, ser parte del progreso tecnológico, científico, social, cultural o económico y así aumentar o asegurar el bienestar de sus ciudadanos, semejante nación debe ofrecer una perspectiva futura y debe medirse y competir internacionalmente. El reclamo de particularidades y excepciones es hoy más bien un pretexto para justificar carencias o la necesidad de enfrentarse a ese desafío.

El desafío hoy es aún mayor que el de la Revolución Industrial del siglo XIX, ya que no se trata de dominar un mundo mecánico, sino un mundo digital, virtual que exige un conocimiento que se origina en la investigación de base, que implica una enorme y constante inversión y tener un grupo de cabezas de primera línea.

Hoy vivimos, además, en una revolución científico-tecnológica permanente cuya trascendencia es muchísimo mayor que la del siglo XIX, y menos perceptible por su forma casi invisible de producción. Se trata de una revolución invisible y limpia. El Web es, espacialmente visto, infinitamente mayor en su efecto que una industria de 
automóviles, pero no ocupa ningún lugar topográfico.

En Latinoamérica se habló, discutió, pensó y escribió mucho sobre la famosa modernización, un debate que ha sido tan ideológicamente marcado y que quizás por ello no logró los resultados perseguidos, y no tuvo una inserción efectiva en la práctica.

\section{La sociedad del conocimiento / ser visible y perceptible internacionalmente}

El mundo actual exige una sociedad de conocimiento, exige una gran inversión en la educación, pero de un tipo determinado, especialmente eficaz y competitivo. Esto significa que su producción, cualquiera que ésta sea, debe ser visible y perceptible internacionalmente. En torno a esta premisa se debe reorganizar la educación. Una producción con meros efectos locales es un despilfarro de recursos económicos y humanos. Al fin, las Humanidades como las Ciencias Sociales deben ser evaluadas como las Ciencias Naturales- basándose en el valor, la resonancia y el efecto de la producción de conocimiento.

\section{Economización, funcionalización y pragmatización de la educación}

Estamos viviendo una fuerte economización de la cultura y de la educación. Existe una evidente funcionalización y pragmatización de la misma. El reto no consiste en resistir estoicamente reclamando criterios humanistas, sino en legitimar la necesidad del conocimiento, de la cultura, de las Humanidades y esto se puede hacer solamente si se entra en el debate de la economización y funcionalización.

El Humanismo fue en su tiempo el sistema de pensamiento más innovador y visionario, no fue ni defensivo ni conservador, fue altamente progresista y de vanguardia. La pregunta básica por responder es: ¿para qué está una disciplina determinada, en qué se basa su legitimación de ser subvencionada por dinero público o privado? ¿Cuál es su derecho de ser? El mayor peligro de la universidad como un centro de educación y ciencia en este debate es la mercantilización o subordinación de la cultura y de la educación a las necesidades del mercado. Al Humanismo renacentista y dieciochesco le siguió el nuevo (o neo) Humanismo decimonónico representado por Humboldt, Fichte y Hegel-ya preparado anteriormente por Herder, Goethe, Schelling y Schleiermacher-, que lleva a una verdadera revolución del sistema educacional en Europa del XIX y que tuvo casi doscientos años de vigencia. Por ello, creo que a pesar de todos los defectos del actual bachelor y del master, la intención que llevó a este cambio tiene una buena cantidad de semejanzas con lo que Humboldt emprendió y que fue paulatinamente estropeado: la reconciliación entre ciencia, educación y práctica que se refleja en el actual sistema en la introducción las "cualificaciones básicas" ("Schlüsselqualifikationen")

\section{4. "Las dos culturas"}

Desde el siglo XIX y a través de todo el siglo XX se viene dando una batalla entre las Ciencias Naturales y de Ingeniería, y las Humanidades y de las Ciencias Sociales, con respecto a su importancia y relevancia y, al parecer, a desmedro de las últimas, 
ya que las Matemáticas, por ejemplo, tienen ya una prioridad absoluta, así por ejemplo, en la escuela primaria en Alemania. El mito de estas "dos culturas", como lo postula Charles Percy Snow en 1959 (vid. Frühwald 1991/21996, 23-24) obedeciendo a un positivismo simplista, ha sido contraproductivo ya que ha llevado a una confrontación de tipo utilitarista demostrándose así más bien la incapacidad de aceptar diversos tipos de ciencia (ibíd., 15-16).

Sin embargo, las Humanidades y las Ciencias Sociales, particularmente las primeras, gozan de una larga tradición epistemológica, son capaces de abarcar la descripción e interpretación de diversas culturas, sistemas culturales, de trabajo y de vida "incluyendo el desarrollo de las Ciencias Naturales, [que] se refieren en un proceso hermenéutico y explicativo, en un acto de ilustración y de emancipación a la forma (aspecto) cultural del mundo" (Frühwald, ibíd.: 16). Negarles a las Humanidades la producción de conocimiento - de un conocimiento con otra utilidad que el de las Ciencias Naturales-es uno de los mayores errores, de los más divulgados y aún actuales en la discusión pública. Por ello, una tarea primordial del debate sobre la reforma educacional debe llevar a la superación de esta dicotomía, al menos a un nivel políticoeducacional, ya que tiene serias consecuencias para el apoyo económico de y para el trato que se les da a las Humanidades y a las Ciencias Sociales en las universidades y en la sociedad. No se trata de reconciliar las diferencias entre las Humanidades y las Ciencias Sociales, sino simplemente de aceptar una recíproca legitimidad.

Se trata de despertar el interés de moverse dentro de otras disciplinas y de comprender que las Humanidades y las Ciencias Naturales no están divididas ni representan polos intransmisibles, como lo demuestran las novelas de Jules Verne, toda la literatura de Science Fiction y muchos textos de Vannevar Bush, Alan Turing, Claude Berger o de J. L. Borges, como veremos más adelante. El debate sobre las células madres es largo que no solamente incluye la biotecnología, sino la ética, la teología, la teoría de la cultura, la política, etc.

\section{Descentralización}

El mundo global exige además una descentralización de los lugares de producción y de las formas de producción y una descentralización de las instituciones.

\section{Educación para la mayoría}

Estas premisas implican además que un país debe dar educación a la gran mayoría de su población. Excluir grandes sectores de la educación es un daño económico, tecnológico y social inaceptable, ya que una población educada, es una población laboral, es, por ende, una población consumidora, una población de contribuyentes, en fin, una población democrática, considerando que la gran parte de los conflictos sociales y políticos provienen de la desigualdad. La exclusión de grandes sectores obedece más bien a un concepto arcaico de una sociedad y no obedece ni al principio de una sociedad civil ni al de una sociedad con principios cristianos. Permítaseme dar algunos ejemplos de Alemania, país que conozco mejor, no obstante, estas observaciones también son válidas para Europa Occidental. A pesar de todas las 
dificultades económicas, de las diferencias sociales cada vez mayores y graves, Alemania dispone de un sistema de educación, de salud y de servicios para toda la sociedad. Las puertas están abiertas a todos los sectores sociales y el Estado tiene una obligación ética de apoyar en particular a los sectores más débiles. Existe una profunda conciencia social en el país. Por ello, Gerhard Schöder pudo ser abogado y canciller de Alemania, a pesar de venir de una familia modestísima: el padre obrero, muerto en la Segunda Guerra Mundial y su madre empleada. El hermano de Schröder es alcantarillero. Schöder comienza como trabajador asistente de construcción, luego pasa a ser empleado comercial y asiste paralelamente al colegio medio, cursa el bachillerato en la escuela nocturna, para finalmente estudiar derecho. Franz Joseph Strauß, el famoso político de la Unión Social Cristiana, era hijo de un carnicero y asiste nada menos que al Maximiliansgymnasium en Múnich, un colegio de elite, para luego estudiar Humanidades. Estudia Filología Antigua (Griego y Latín), Literatura Alemana, Historia y Ciencias de Estado y fue becario de la Stiftung Maximilianeum, una de las fundaciones más prestigiadas de Alemania. Willi Brandt, el ícono de la política alemana y europea, es otro caso típico para una sociedad moderna: su madre fue vendedora de negocio, él fue hijo ilegítimo, hizo su bachillerato, pero ni estudió ni tuvo educación profesional alguna. A este tema volveré más adelante. Naturalmente que estas personas pertenecen a otra generación, pero cada época tiene sus ventajas y dificultades.

Volviendo a las seis premisas enunciadas, éstas deciden y determinan la línea de demarcación de si un país es parte del tercer mundo, de un mundo en vías de desarrollo o del primer mundo. El desperdicio de recursos y potenciales humanos es una ceguera que ha transformado al subdesarrollo en algo crónico, en una especie de maldición, de destino inevitable. Esto no debería ser así, ya que es más bien el resultado de la incapacidad de los actores políticos, científicos, educacionales, económicos y culturales de ponerse de acuerdo sobre una agenda que solucione los problemas como lo demuestra la coalición entre el SPD (Partido Social Demócrata), el CDU (Unión Democrática Alemana) que han solucionado una serie de problemas fundamentales en menos de dos años, y que estaban acumulados hace más de veinte.

Para enfrentar el problema en nuestro campo concreto comienzo con la pregunta ¿cuál es el estado actual de las Humanidades y de las Ciencias Sociales? (Me referiré en particular a las Humanidades.)

En relación con el primer aspecto, ¿cuál es el estado actual de las Humanidades?, podemos constatar que:

- han perdido o están perdiendo su reputación tradicional,

- han perdido o están perdiendo su autonomía,

- la reformulación de la disciplina ha conducido a una función subalterna y de disciplinas auxiliares o de servicio para disciplinas tales como la Economía y las Ciencias Exactas.

Las Humanidades en general, pero en particular las Ciencias Literarias, han perdido gran parte de la fascinación y relevancia sociopolítica de la que gozaban en los años sesenta y setenta, hasta más tardar la mitad de los ochenta. Esto también 
ocurre en Alemania, a pesar del prestigio del que aún gozan la institución 'universidad' y las Humanidades en el país.

Ello se ve claramente cuando se discute sobre la tecnología genética o sobre la investigación en los campos de la cognición, del cerebro y del lenguaje (Kognitions-/ Gehirn- und Sprachforschung), donde las Humanidades son siempre auxiliares y cuando las personas involucradas tienen una competencia científica en el campo de la medicina. Las disciplinas que se salvan de no ser descartadas son la Sociología, la Filosofía o la Lingüística cuando tienen una orientación dentro de las Ciencias Naturales. De allí se desprende la pregunta fundamental de para qué estamos y quién nos necesita, ya que en la discusión pública los Estudios Culturales -que están muy de moda hoy en día-casi no tienen ninguna inserción y el aporte de los estudios de literatura al discurso social es inexistente en estos debates. Esto se ve también en que la agobiante mayoría de programas de investigación subvencionados por instituciones privadas o estatales o las prioridades del aparato gobernante de las universidades favorecen a las Ciencias Sociales y Naturales, aquí a los campos ya mencionados, más la Biodiversidad, la Biotecnología, las Matemáticas y no a las carreras de Humanidades.

En el periódico Die Welt del miércoles 22 de agosto de 2007 se constata que en los últimos años se han cancelado 1458 cátedras (Full Professorships) y en particular en las Humanidades y en las Ciencias Sociales (una aseveración que contradicen algunos sectores provenientes de las Ciencias Exactas que afirman que las Humanidades y las Ciencias Sociales no han sufrido más que las Ciencias Exactas, en muchos casos se sostiene que las Humanidades y las Ciencias Sociales han obtenido un alza de las cátedras, particularmente en los Estudios Culturales.

El problema tiene que ver, además, con la reflexión sobre una redefinición o reestructuración en particular de las Humanidades y del campo de la Crítica Literaria (CL) académica en relación con los Estudios Culturales (EC) que es un fenómeno global y que se refleja cuando, por ejemplo, se pregunta ¿cómo reaccionar frente a la disminución de estudiantes subgraduados y graduados de Literatura y al incremento de los abocados a los Estudios Culturales?

Esta pregunta revela un fenómeno que se está dando masivamente en todos aquellos países en cuyas universidades se introdujeron los Estudios Culturales y en las que se están redefiniendo las disciplinas de las Humanidades.

El problema tiene que ver con la idea que tenemos de lo que es la universidad y cuáles son las tareas en la investigación o en la docencia, problema que se ha agudizado, por ejemplo, en Alemania, con la distinción de universidades de investigación, esto es, de elite (diez universidades fueron elegidas por la Deutsche Forschungsgemeinschaft (German Counsil of Research)) y de universidades de enseñanza. Para mí, personalmente, y esta es la posición de la Universidad de Leipzig que se refleja también en la estructura del Bachelor y del Master, la universidad es primordialmente un lugar de investigación de donde la docencia es un resultado de la investigación (y no al revés).

En Inglaterra, hace decenios, los estudios literarios y culturales son absolutamente 
subalternos (con excepciones en algunos nichos de excelencia), en Escandinavia los profesores comienzan a abandonar la universidad. Allí comienzan a regir en algunos lugares managers con experiencia en Shangai, que han reducido o eliminado los derechos de los órganos académicos; a los profesores que osan manifestarse públicamente en contra de los cambios negativos para la disciplina y la falta de democracia, los amenazan con despedirlos, a pesar de ser funcionarios del Estado. En Italia y España nuestras disciplinas tienen un lugar más que periférico e incluso en Francia la situación es muy precaria fuera de los grandes écoles. Lo que es válido es el Certificat d'aptitude au professorat de l'enseignement du second degré (Capes) en las Ciencias Exactas.

Otro problema que estamos enfrentando es la forma como se define la excelencia académica y constatamos que ésta se define, una vez más, como en el debate de la pluralidad metodológica de los años setenta, basándose en criterios que vienen de las Ciencias Exactas o que las favorecen, esto es, excelencia se define sobre la base de proyectos financiados por instituciones externas (Drittmittel), por publicaciones en revistas indexadas -que son en nuestro campo casi inexistentes-o por la construcción de colegios de doctorados. Publicaciones en una buena revista o colección científica y con peer reviewed juegan un rol parcial y periférico si no son órganos de publicación británicos o estadounidenses, el resto parece no existir, lo cual representa una arbitrariedad sin parangón. Como los criterios de la práctica de otorgar una subvención tienen una base en las Ciencias Exactas es más difícil para las Humanidades y las Ciencias Sociales obtener semejantes subvenciones.

Existe, además, otro aspecto que nos desfavorece: la guerra ideológica, la discriminación, la falta de tolerancia intelectual, académica y científica, el dogmatismo que convierten a las Humanidades y a las Ciencias Sociales en verdaderos campos de batalla y en instrumentos destructores del campo con efectos nefastos, ya que estas disciplinas quedan relegadas a la periferia, como se puede demostrar claramente en el debate sobre la postmodernidad y postcolonialidad o sobre la hibridez que fueron rechazados y combatidos antes de que se hubiesen leído las obras fundamentales del campo. La ideología y la falta de tolerancia es un factor importante que ha contribuido a disminuir la reputación y la importancia de las Humanidades y de las Ciencias Sociales. Este procedimiento ha sido una efectiva manera de periferizarse a sí mismo y, lo peor, es que esto se hace a costa de los jóvenes estudiantes y futuras generaciones. El sectarismo académico en la universidad, particularmente en las Humanidades y las Ciencias Sociales es una verdadera plaga que nada tiene que ver con un espíritu crítico. Pero dentro de un sistema plural se debe tener la tolerancia de las diversas aproximaciones sin caer en fundamentalismos ideológicos. Las diversas teorías no son instrumentos de exclusión y discriminación, sino más bien caminos complementarios.

Mi conclusión es que las universidades deben desarrollar una estrategia para reclamar los derechos y necesidades de las disciplinas no "exactas", para lo cual, si queremos mantener el estatus actual, se deberían considerar los siguientes aspectos. 


\section{Teorías y metodologías fuertes}

Debemos formular la disciplina de las Humanidades sobre la base de teorías y metodologías que merezcan este nombre (hoy en día existe una carencia crónica de bases metodológicas y esto no es el resultado de la postmodernidad que tiene bases teóricas muy fuertes, sino más bien un resultado del agotamiento de la discusión teórica de los setenta y ochenta que cayó, a mi modo de ver, en un callejón sin salida).

\section{Globalización de la teoría}

Fuera de eso se debería superar el prejuicio de distinguir entre teorías que vienen de afuera o aquellas de origen local, que es un arma o una forma de proteger lo conocido y de evitar la innovación y que tiene el fatal efecto de petrificar el retraso (a este punto volveré a continuación), lo cual ha relegado a Latinoamérica a una especie de subdesarrollo elegido. Se necesita una globalización en la teoría como es el caso en las Ciencias Exactas.

\section{Establecimiento de estándares de excelencia}

Se deben establecer estándares de excelencia (que es un problema arduo de definir) de tal forma que programas académicos, proyectos científicos y publicaciones sean capaces de competir internacionalmente. $Y$ es exactamente en este aspecto donde se puede constatar que publicaciones que tienen una base teórica fuerte, son las más competitivas y las más ampliamente recibidas. Con respecto a Latinoamérica, los proyectos no pueden ser aceptados o rechazados según los méritos académicos ya obtenidos o según la trayectoria académica exitosa o no de la persona concursante basándose en juicios favorables dados a priori por personas cercanas al concursante, los criterios de selección deben basarse solamente en criterios generales de excelencia y deben depender de la calidad del proyecto propuesto.

\section{Relación discurso teórico e instituciones}

Nuestra disciplina debe tener o al menos buscar una inserción en los procesos sociales o socioculturales, esto es, debe tener una presencia en las instituciones.

\section{Facilitación de habilidades}

Las Humanidades y las Ciencias Sociales no tienen ni deben tener la finalidad de preparar para un oficio determinado (no son berufsqualifizierend), pero sí deben ser capaces de ofrecer habilidades determinadas que puedan tener un amplio uso en el mundo profesional. Lo que no podemos tener en todas partes son ciencias aplicadas. Si la universidad no es capaz de formular la predominancia de la investigación se convertirá en una escuela técnica superior o politécnico (Fachhochschule) que resuelven sus tareas en forma tan excelente que no necesitan una duplicación.

Esto implica que debemos definir el tipo de universidades que se quiera tener y cada una debe tener su función y su perfil. Estoy hablando de universidad en el sentido clásico: de una institución que tiene todas las facultades y de una institución donde la investigación es lo principal y sus resultados desembocan en la enseñanza y, en algunos casos, en labores prácticas. 


\section{REORGANIZACIÓN DE LA INSTITUCIÓN UNIVERSIDAD}

\subsection{Transdisciplinaridad}

Un primer paso es organizar las Humanidades y las Ciencias Sociales en una forma transdisciplinaria. Esto requiere un nuevo concepto de organización científica y una nueva forma institucional.

El prefijo 'trans' representa una ampliación y deslimitación de aquellos con el prefijo 'inter' ya que se ubica en un supranivel epistemológico. Pero a la vez implica que sin un módulo-núcleo en un campo determinado (disciplina), cualquier tipo de construcción 'transdisciplinaria' es imposible. El término 'trans' exige una disciplina entrelazada en una red de parámetros. Se trata de un diálogo concentrado en objetos o preguntas comunes.

Por 'transdisciplinariedad' entiendo, por una parte, el recurso a modelos de diversa proveniencia disciplinaria y teórica (p.e., teatral, histórica, antropológica, sociológica, filosófica, estructural, postestructural, teoría de la comunicación), el recurso a unidades o elementos particulares de éstos al servicio de la apropiación, decodificación e interpretación del objeto analizado. El término de 'transdisciplinariedad' tiene poco que ver con aquel tradicional de la comparatística e interdisciplinariedad ya que allí los métodos de la propia disciplina de base no se trascienden. La 'transdisciplinariedad', más bien, incluye y conlleva diversas aproximaciones, ya que el empleo de postulados e instrumentos de otras disciplinas implica siempre una deterritorialización y reterritorialización de éstas.

'Transdisciplinariedad' no es un eufemismo para hablar del "fin de la ciencia" ni para hablar de la pérdida de la unidad y rigurosidad científica, no representa la imposibilidad de pensar las Humanidades dentro de un 'sistema científico' (vid. más abajo), sino que apunta hacia un nuevo tipo de organización científica entre las disciplinas, disciplinas entrelazadas recíprocamente (vid. Frühwald 1991/21996, 16).

Se debería repensar la organización de la universidad en las Humanidades y Ciencias Sociales, actualmente agrupadas en facultades y departamentos autónomos que en algunos casos, y no los menos, son más bien feudos manejados por personas que están hace decenios en el mismo puesto y en muchos casos haciendo y diciendo lo mismo. Más bien, estos modelos deben pasar a ser módulos logísticos de una disciplina dentro de una estructura superior donde la enseñanza modular sea transinstitucional y transdisciplinaria, algo que también se puede lograr sobre la base de la creación de Bachelors o Másters con módulos de diversa proveniencia.

Los departamentos o las agendas curriculares deberían ser transformados en perfiles o módulos donde diversas disciplinas intervengan. Por ejemplo, pregunto ¿es posible ocuparse de la época del Descubrimiento, Conquista y Colonia de América solamente bajo un punto de vista literario? ¡Naturalmente que no! Aquí se requiere la intervención de historiadores, de teóricos de la historia y de la cultura, de etnólogos, arqueólogos, antropólogos y expertos en lenguas precolombinas, etc.

\subsection{Didáctica}

Los cursos deberían impartirse, desde el comienzo, en forma de seminarios donde 
los estudiantes puedan participar activamente. Fatal es la aún muy difundida enseñaza que llamo frontal, donde el profesor habla y el alumno toma apuntes que luego deberán ser reproducidos en un examen escrito. Aquí se sacrifica el desarrollo de la autonomía del estudiante, el estímulo de su iniciativa, su creatividad y su espíritu crítico.

La experiencia de los estudiantes con este tipo de didáctica, como se da en Latinoamérica, Italia, España y Francia, es más que negativa. Con la introducción del Bachelor y del Máster se corre el peligro de que este tipo de enseñanza se propague aún más, ya que la mayoría de los cursos se clausuran con una examen escrito ${ }^{3}$.

La prioridad de nuestra tarea pedagógica no debería ser o no se debería agotar en la transmisión de un conocimiento, el conocimiento se encuentra en las bibliotecas y en forma masiva; nuestra tarea debe consistir en desarrollar la importante facultad de pensar y desarrollar habilidades, que es lo que le da la autonomía al estudiante y la capacidad de decisión como futuro profesional, y asimismo le da la legitimación a las Humanidades y a las Ciencias Sociales, y a la universidad ya que esta capacidad sólo, o particularmente, se puede fomentar en las universidades y dentro de las Ciencias Sociales. Naturalmente que una didáctica de este tipo debe comenzar en la escuela primaria como es el caso en muchos países; mas este es otro tema, pero muy ligado a la educación superior, ya que la primaria es, quizás, el momento más importante en la vida de futuros alumnos, allí se ponen las bases de todo lo que está por venir.

Conocimiento-saber/pensar/habilidades: ésta es la fórmula para el presente y para el futuro. Con esta fórmula apunto naturalmente al principio universitario de Humboldt, donde a los estudiantes no solamente se les debería transmitir un conocimiento, sino también "cualificaciones básicas" ("Schlüsselqualifikationen", un término central en la actual reforma curricular) que son habilidades intelectuales, científicas y sociales.

La unidad de 'ciencia-investigación y docencia' debe colocarse en el centro de las nuevas reflexiones y no debe sustituirse por la fórmula de 'ciencia y práctica' que conduciría a un empobrecimiento de las Humanidades y de las Ciencias Sociales, lo cual en muchos casos ya ocurre. Por el contrario, lo que debe existir es una estrecha relación entre ambas fórmulas ${ }^{4}$.

La división entre investigación y docencia marca el fin no sólo de las Humanidades, sino también de la universidad. La aplicación de teorías implica una investigación base y por ello la investigación debe ser independiente de finalidades puramente utilitaristas. Además, y volviendo a un punto ya indicado más arriba, la división alemana en universidad, escuela técnica superior (o politécnico) y en universidad técnica es una estructura que debería mantenerse. Pero la universidad

\footnotetext{
3 Por el contrario, en el Instituto de Romanística de la Universidad de Leipzig se estableció como principio de calificación de curso una presentación oral y una tesina al fin del curso.

4 La estrecha relación entre investigación y docencia que ha existido en el Instituto de Romanística de la Universidad de Leipzig, al menos desde 1993, se conservó en el nuevo currículum y no sucumbió a un tipo de enseñanza repetitiva, mimética y meramente mecánica.
} 
ha sido, a raíz de la presión política, cada vez más transformada en los dos últimos decenios en una especie de politécnico.

Si las disciplinas en cuestión pueden hacer una contribución importante, es precisamente poner al estudiante en una situación de poder elegir y organizar su material en forma autónoma, bajo la tutoría del profesor, se entiende.

Un conocimiento puramente mimético es un no-conocimiento, ya que no conduce a hacer pensar a los estudiantes, y ésta es una habilidad que no es dada de antemano, sino que debe fomentarse a través del ejercicio. Por ello los cursos deberían organizarse basándose en unidades didácticas, con un tema monográfico y un corpus determinado donde los estudiantes deban hacer una presentación oral y al fin del curso escribir una tesina.

Quisiera también ilustrar lo expuesto con ejemplos concretos. Hoy en día, las compañías, particularmente en el mundo de los servicios, no requieren necesariamente expertos en algún campo, sino que más bien exigen, en primer lugar, jóvenes con flexibilidad, creatividad y eficacia intelectual; en segundo, personalidades bien perfiladas; en tercero, experiencia en el extranjero y, en cuarto, lenguas. ¿Y qué disciplinas sino las Humanidades en particular pueden impartir semejantes bases?

Los políticos que mencioné más arriba desarrollaron diversas habilidades que les permitieron ocupar los cargos para los cuales fueron elegidos. Strauß era, por ejemplo, reconocido mundialmente por su enorme saber y competencia en la economía, pero también en un cúmulo de otros campos. Michael Glos, el actual Ministro de Economía, asistió solamente al colegio medio y es molinero de profesión; el jefe del Estado de Rheinland Wesfalen, Kurt Beck, es electricista de profesión. Varias de mis estudiantes ejercen profesiones en otros campos que el de la Filología: una de ellas es manager en la famosa compañía de cubiertos de plata Robbe \& Berking en Flensburgo, otra es manager en una sucursal de la Volkswagen, otra es periodista en el famoso y elitista periódico Die Zeit, sin haber estudiado periodismo. Y así podría continuar con la lista.

\subsection{Concurso a la cátedra}

Otro aspecto fundamental en parámetros de reforma y de excelencia es el concurso de cátedras. Estos concursos deberían ser internacionales, sobre la base de una comisión transnacional e internacional, donde la persona que obtenga la plaza sea la mejor en su campo y no la persona que le parezca mejor a una persona o a un grupo de catedráticos que están ya hace años en el campo. Oxigenación debe ser el lema y la formulación de las características de una cátedra que se debe definir según parámetros de excelencia y necesidad internacional y no tan sólo por criterios locales.

Se deberían distinguir al menos dos tipos de cátedras: cátedras máximas para super stars y cátedras en un escalón más abajo, cátedras que puedan desarrollarse o ascender en el futuro.

Las cátedras no deben ser de inmediato de por vida, sino que el contrato se debería ir renovando según el rendimiento, lo que implica una evaluación en primer lugar de las publicaciones y resultados científicos, luego de la enseñanza y del trabajo 
administrativo. La permanencia en la cátedra debería ser sustancialmente limitada. Profesores con hasta 72 años sin conexión y tampoco sin las habilidades para entender al mundo actual, tienen poco que decirle a una estudiantado casi cincuenta años más joven.

\subsection{Criterios de excelencia, evaluación}

La definición de los criterios de excelencia es un desiderátum y por ello muy difícil de realizar.

Fundamental es que la comisión de evaluación venga de fuera de la universidad donde se van a evaluar a los profesores. Importante sería que también integrasen una semejante comisión profesores extranjeros. Evaluaciones locales e incluso con miembros de la misma Facultad o del mismo Departamento carecen de sentido.

Naturalmente que en primer lugar se deben evaluar las publicaciones según parámetros de excelencia (peer reviewed, indizadas, prestigio de la editorial y de la revista o colección, recepción y citación de los trabajos, número de publicaciones, etc.). Mientras que esos criterios funcionan ya hace mucho en el mundo de las Ciencias Exactas, no es este precisamente siempre el caso en las Humanidades y Ciencias Sociales, sin embargo, esto comienza también a cambiar, especialmente por la presión de la globalización: la competencia por los buenos alumnos no es ya más algo nacional, sino internacional: el asunto es qué universidades tienen algo que decir en el mundo.

En segundo lugar se debe evaluar la docencia, pero no tan sólo partiendo de criterios estadounidenses, del voto de los estudiantes cuyos juicios son altamente subjetivos y en muchos casos emocionales, sino también partiendo de la forma de organizar los cursos, del material empleado, de la intensidad de la tutoría, etc., y esta evaluación debe a su vez ser realizadas por compañías especializadas y por personas externas.

\subsection{Redefinición de la disciplina en las Humanidades}

Desde el Renacimiento, pero particularmente en la Ilustración y finalmente en la Modernidad, las artes se independizan de la religión y la teología para entrar en una relación entre arte y filosofía, entendida esta última en el sentido de Hegel no solamente como "die eigentliche Führungswissenschaft, sondern die geistige Führungsmacht, die das Ganze zu begreifen und Sinn zu vergegenwärtigen oder zu stiften mit Fug und unbestritten beanspruchen konnte" o según Fichte, Schleiermacher y Humboldt como "reinste Form des Wissens" o "Feld der reinen Wissenschaft (en Frühwald et alii 1991/21996, 82), esto es, como una nueva relación bajo el liderazgo de la filosofía, como ciencia punta y como el lugar de la elaboración de una forma pura del conocimiento y de la ciencia (ibíd., 83).

Hoy nos encontramos, al parecer, en la última fase de la destrucción del sistema universitario de Humboldt, quien entienda la ciencia como una totalidad de disciplinas relacionadas entre sí, la ciencia como integración y como construcción de la personalidad individual, donde la Facultad de Filosofía sea el centro del 
microcosmos científico (ibíd., 85), fase que es el resultado de la demanda que se repite de periodo en periodo y en forma estereotípica de un "racionalismo utilitarista", que exige la utilidad social y económica de las disciplinas y su capacidad de aplicación. Esta exigencia de tipo pragmático-económica si no se resiste, será el fin de las Humanidades y de parte de las Ciencias Sociales ya que esta exigencia pretende documentar la inutilidad de las mismas frente a un cambio de paradigma que va de un desplazamiento de la investigación o ciencias base a la investigación o ciencias aplicadas, donde a nivel industrial se salvan, fuera de la física y la química, hoy en día, la biología (en particular la genética) y las ciencias moleculares (vid. ibíd., 87).

La "crisis" de la universidad es doble: una externa determinada por las exigencias utilitaristas que le pueden hacer perder su autonomía frente al Estado y a la economía, otra interna que consiste en la reformulación de su idiosincrasia.

Estamos viviendo una situación semejante a aquella de los años sesenta donde todo se cuestionó y las Humanidades tuvieron que redefinirse según su importancia social y a través de una fundación teórica ${ }^{5}$.

La diferencia entre ese momento histórico y el actual radica no solamente en que la ideología política no tiene ningún papel en el debate, sino en que las redefiniciones se formulaban dentro de las fronteras de cada disciplina y eran de tipo local. Hoy, una semejante redefinición es mucho más compleja, ya que se debe hacer fuera de las disciplinas tradicionales, en un "tercer espacio nómada" -como quisiera llamarloque se concretiza cada vez según la demanda del objeto, del lugar y de la intención de la enunciación. Cada objeto cultural por tratar, va a exigir su módulo particular de teoría; por otra parte la recodificación de las disciplinas es transnacional, transcontinental, global. Es decir, es imposible que un crítico de literatura (u otro) pretenda hoy redefinir su disciplina dentro de la disciplina misma, sin considerar lo que está pasando en otras disciplinas vecinas y en el resto del mundo. Un procedimiento semejante (y masivamente existente, sin lugar a dudas) conduce al estancamiento. La redefinición o reformulación de la disciplina hoy lleva siempre a una deslimitación o descentración de las fronteras disciplinarias y aquí radica hoy el "cambio de paradigma" fundamental de pensar la disciplina y la teoría (vid. también Hall 1990, 6). Deslimitación no significa fragmentación, arbitrariedad, relativismo, caos o diletantismo, sino entrelazamiento de diversas aproximaciones para dar una visión lo más completa, esto es, lo más abierta posible. Y, del riesgo que conlleva el valerse de diversos fragmentos teóricos productivos se controla a través de una red discursiva, teórica y metodológica, ya que el mero "préstamo" no entrelazado de postulados de disciplinas vecinas sería repetir esa parte de la disciplina sin llegar a una deslimitación.

Una reformulación de la disciplina abarca además un aspecto teórico y otro institucional, al cual me referí más arriba, y otro docente o práctico (estudio de campo,

${ }^{5}$ La discusión actual pertenece al tópico de las famosas polémicas entre antiguos y modernos desde el Renacimiento italiano y francés, revividas en el siglo XIX en Francia en el debate del realismo, o por Borges en sus escritos de los años veinte y treinta (El tamaño de mi esperanza, Inquisiciones, El idioma de los argentinos o en Evaristo Carriego) o por la nueva novela latinoamericana y francesa a partir de los años cincuenta hasta los sesenta, por mencionar tan sólo ejemplos en el campo de la literatura. 
de localización de la teoría y del objeto y su localidad; siendo aquí donde radica el mayor problema).

Una reformulación la basaría en la transdisciplinariedad que incluiría conceptos o estrategias tales como 'hibridez', 'transversalidad', 'transmedialidad' y 'transculturalidad'.

Me parece imprescindible indicar que en la formulación o aplicación de una teoría no es su lugar de origen sino su productividad su capacidad de generar conocimiento lo que vale. Por 'productividad' teórica se pueden entender dos procesos simultáneos: la potencialidad de explicación que nos ofrece el instrumentario teórico elegido y su recodificación dentro del lugar geopolítico de aplicación con respecto al objeto a tratar.

Si el objeto cultural contiene tanto elementos locales como translocales, la teoría podrá difícilmente operar de otra forma. La cultura (o una buena parte de ella), al mismo tiempo, ha operado siempre de una forma transcultural y ajerárquica; se podría sostener que cultura o literatura se pueden definir por esta transculturalidad, descentración, nomadismo y por habitar muchos espacios a la vez, una evidencia, por lo demás.

Para evitar una buena cantidad de problemas especialmente ideológicos, y en menor grado teóricos, me parece más adecuado ya no hablar de Estudios Culturales (EC), sino más bien de una Teoría de la Cultura (TC) -basada en un tipo de teoría transversal o híbrida-, ya que este término está fuera de cualquier pertenencia, no está centrado en un espacio geopolítico-académico determinado ni tampoco está caracterizado por un canon de ciertos temas y focos de investigación y ofrece, por esto, una mayor amplitud ${ }^{6}$. Fuera de eso, una teoría de la cultura se relacionaría más fácilmente con otras disciplinas.

La necesidad de formular una TC en general, y en particular para Latinoamérica, no radica en una cuestión de coyuntura (moda) teórico-académica o en la necesidad de supervivencia de una disciplina, sino en el objeto mismo, en la cultura que exige nuevas aproximaciones.

Una construcción teórica va a tener naturalmente consecuencias tanto para la organización institucional como para el currículum. El problema por resolver radica en saber hasta qué punto es posible, necesario y productivo conservar una especificidad y hasta qué punto es manejable una deslimitación del campo. Por esto, una reflexión sobre los EC o sobre la Crítica Literaria (CL) implica que ambos deben ser reformulados y no uno en contra del otro. Tanto los EC como la CL se reunirían en una TC.

Para mi propósito de proponer como base la 'transdisciplinariedad' para la reformulación de las Humanidades y de las Ciencias Sociales, parto de la concepción o de la construcción de disciplinas transversales (término que tomo de Deleuze), esto es, de disciplinas entrelazadas, interrelacionales y dependientes unas de las otras.

6 Hall (1990: 16) parece nivelar el término 'cultural studies' con el de 'cultural theory', no así con el de 'theory of culture', sin embargo, quisiera entender estos dos últimos términos como sinónimos. 
Hoy, el tipo de problemas a tratar sobrepasa el tipo de preguntas que constituían tradicionalmente las Humanidades, o formulado de otra forma, hoy se incluyen campos y preguntas que antes quedaban excluidas, por ello, por ejemplo, la literatura, como disciplina, debe ser considerada como módulos o núcleos de concentración científica dentro de un sistema transversal de ciencia. Esto es, el establecer una red de aspectos fundamentales de campos y preguntas para describir y comprender los discursos actuales o del pasado. El objeto cultural (por ejemplo un texto) se debería entender como una unidad significativa, como un material y punto de partida dentro de una red de comunicación ${ }^{7}$.

Así, en vez de una competencia disciplinaria los estudiantes tendrían una competencia discursiva transdisciplinaria. De esta forma se cuestionarían las hegemonías disciplinarias o teóricas. La CL sería un núcleo y punto de partida vital, porque el así llamado discurso ficcional ("literatura") sería producto de una red de diversos discursos y conlleva un conocimiento y una verdad por transmitir. Por esto, la estructura y localidad y las convergencias de la CL con los EC y con otras disciplinas resultan de preguntas apremiantes en el mundo. De allí que el mero cuestionamiento del estatus de la CL es una pregunta que parte de postulados equivocados. La pregunta es, ¿qué aprendemos de la literatura, histórica, social, política y epistemológicamente hablando?

La TC sería pues un lugar privilegiado de análisis, de descripción y de interpretación de las construcciones culturales y sus diversas articulaciones o representaciones en sistemas discursivos.

La TC representa una necesidad y una alternativa al derrumbamiento de la coherencia metodológica de las disciplinas a partir de los años ochenta, que conlleva a una fuerte especialización y fragmentación del conocimiento. La TC se presenta como el lugar que da cohesión teórica a las Humanidades y a las Ciencias Sociales sin caer en los dogmatismos de los decenios anteriores. La TC tendría, además, la tarea de superar la división entre ciencia/saber/conocimiento y sociedad, entre educación y sociedad y así legitimar su acción sin tener que obedecer en cualquier caso a los principios de mercado. El empobrecimiento de la universidad, en particular de las Humanidades, su pérdida de prestigio y de legitimación radica, según Habermas (1981, II, 488), por una parte, en la falta de marcos y contextos con claros conceptos de ciencia y enseñanza, en una fuerte reorganización de las universidades y del saber en torno a culturas de expertos sin una práctica o relación a procesos sociales cotidianos. Es decir, un falso concepto de elite conlleva a una aislamiento de cierto tipo de universidades y a la masificación de otras.

Una TC tendría como tarea, pues, detectar campos de intereses comunes entre las Humanidades, las Ciencias Sociales y las Ciencias Naturales. La selección del siglo XIX que reduce a las Ciencias Naturales a lo meramente empírico sin considerar

\footnotetext{
7 Ya muy pronto Richard Johnson (1986/1987: 62) aboga por una descentración del objeto (texto) como objeto inmanente de estudio:

"The text « is no longer studied for ist own sake, nor even for the social effects it may be thought to produce, but rather for the subjective or cultural forms which it realises and makes avaulable. The text is only $a$ means in cultural studies; strictly, perhaps, it is a raw material from which certain forms [...] may be abstracted. It may also form part of a large disvursive field or combination of forms occurring in other sozial spaces with some regularity.
} 
los elementos sociales, psicológicos y culturales y viceversa, la definición de las Humanidades y de las Ciencias Sociales como ciencias hermenéuticas de corte filológico, condujeron a una reducción y división de estas "dos culturas", de las Humanidades y las Ciencias Sociales por un lado y de las Ciencias Exactas por otro.

Resumiendo: la TC se presenta como la instancia que le puede devolver la legitimidad a las Humanidades en cuanto:

a) es el lugar donde se reflexionan, se comunican y se explican los problemas civilizadores, por ejemplo, hoy en día, de la globalización;

b) tiene su origen y su función en las intersecciones de épocas, de culturas y de ciencias; se encuentra en las cesuras de articulación entre tradición e innovación y es siempre de carácter ilustrativo;

c) es la instancia o el lugar privilegiado de la crítica y del cuestionamiento;

d) es la instancia de la libertad de pensamiento y en donde se tratan todo tipo de problemas racionalizados o tabúes, en donde se formulan todo tipo de preguntas;

e) es la instancia de la traducción y mediación del pensamiento y de los discursos científicos frente a la sociedad;

f) se configura como discurso de emancipación, productor de un espíritu civil y democrático.

Las Humanidades y las Ciencias Sociales son el lugar en el cual las sociedades modernas construyen un saber sobre sí mismos en forma científica y cuya función es orientadora.

\section{SABER Y CONOCIMIENTO DE LA LITERATURA}

Precisamente por esa libertad de reflexión y de experimento que caracteriza especialmente a las Humanidades, quisiera a continuación dar un ejemplo, entre otros muchos, que pone en evidencia el saber y la necesidad de la cultura y literatura y de su interpretación y permeabilización a través de las Humanidades. Tomo un ejemplo de un autor archiconocido, de tal forma que cualquier lector o receptor podrá posicionarse. Naturalmente que no intento que mi aproximación sea compartida por todos, más importante es el mecanismo que una aproximación pone en marcha.

Para comenzar con estos ejemplos, es importante acentuar el conocimiento o el saber que conllevan objetos culturales, por ello, una división en el nivel del conocimiento entre, por ejemplo, textos científicos y textos ficcionales me parece obsoleta y contraproductiva. Un enorme número de obras centrales de diverso origen recurren al arte o a la literatura para desarrollar sus premisas científicas, como por ejemplo las "escuelas" de la historiogafía de Le Goff y de Hayden White.

\subsection{Borges y la construcción del pensamiento postmoderno}

Permítaseme en este lugar dar algunos ejemplos famosos que demuestran el poder de conocimiento de las Humanidades. Comienzo por recordar a François Lyotard, filósofo francés, a quien el gobierno de Québéc le encomendó la tarea de 
hacer un diagnóstico del estado de la sociedad canadiense. El resultado fue su libro La condition postmoderne (1979), que provocó una discusión mundial que incluso dura hasta hoy.

Otro es Michel Foucault, uno de los filósofos más importantes del siglo XX, quien ni más ni menos describe en su libro Les mots et le choses (1966) los dos grandes cambios de paradigma en el pensamiento y saber del mundo occidental, y que él ubica entre el paso del siglo XV al XVI y del XVIII al XIX. En esta obra, considerada una de las obras capitales de la filosofía y pensamiento occidental, Foucault recurre a un texto de Jorge Luis Borges, "El idioma analítico de John Wilkins", donde éste da una clasificación de la categoría 'mamíferos' en una enciclopedia china que se encuentra en este relato. Este pasaje constituye el punto de partida del famoso libro de Foucault. El conocido fragmento es el siguiente:

En sus remotas páginas está escrito que los animales se dividen en (a) pertenecientes al Emperador, (b) embalsamados, (c) amaestrados, (d) lechones, (e) sirenas, (f) fabulosos, (g) perros sueltos, (h) incluidos en esta clasificación, (i) que se agitan como locos, (j) innumerables, (k) dibujados con un pincel finísimo de pelo de camello, (l) etcétera, $(\mathrm{m})$ que acaban de romper el jarrón, (n) que de lejos parecen moscas ("El idioma analítico de John Wilkins", en: OC $1972 /{ }^{2} 1989$, I, 708).

Foucault lo comenta como sigue:

Dans l'émerveillement de cette taxinomie [d'une certaine encyclopédie chinoise citée par [Borges], ce qu'on rejoint d'un bond, ce qui, à la faveur de l'apologue, nous est indiqué: l'impossibilité nue de penser cela.

[...]

La monstruosité ici n'altère aucun corps réel, ne modifie en rien le bestiaire de l'imagination; elle ne se cache dans la profondeur d'aucune pouvoir étrange. Dans l'émerveillement de cette taxinomie [d'une certaine encyclopédie chinoise citée par [Borges], ce qu'on rejoint d'un bond, ce qui, à la faveur de l'apologue, nous est indiqué: l'impossibilité nue de penser cela.

$[\ldots]$

Ce qui transgresse toute imagination, toute pensée possible, c'est simplement la série alphabétique $(a, b, c, d)$ qui lie à toutes les autres chacune de ces catégories.

[...]

La monstruosité que Borges fait circuler dans son énumération consiste au contraire en ceci que l'espace commun des rencontres s'y trouve lui-même ruiné. Ce qui est impossible, ce n'est pas le voisinage des choses, c'est le site lui-même où elles pourraient voisiner. Les animaux [...] où pourraient-ils jamais se rencontrer, sauf dans la page qui la transcrit? Où peuvent-ils se juxtaposer sinon dans le non-lieu du langage? mais celui-ci, en les déployant, n'ouvre jamais qu'un espace impensable.

[...] 
[...] ce serait le désordre qui fait scintiller les fragments d'un grand nombre d'ordres possibles dans la dimension, sans loi ni géométrie, de l'hétéroclite; et il faut entendre ce mot au plus près de son étymologie: les choses y sont «couchées», «posées», «disposées» dans des sites à ce point différent qu'il est impossible de trouver pour eux un espace d'accueil, de définir au-dessous des uns et des autres un lieu commun. (Foucault 1966, 7-9)

Esta observación fue, como luego concluí, la primera indicación de lo que yo, más tarde, partiendo de Baudrillard, otro fundamental y gran filósofo y sociólogo francés que también recurre a Borges para su teoría de la simulación, había de denominar la virtualidad de la escritura de Borges.

La monstruosidad de la taxonomía es, además, esa estructura que Deleuze y Guattari llamaron "milles plateaux" o "rizoma" y que a partir de 1992 he reflexionado sobre su origen, definición y aplicación, que encontré en los relatos de Borges tales como "Tlön, Uqbar, Orbis Tertius" (1940), "El jardín de senderos que se bifurcan" (1941), “El idioma analítico de John Wilkins" (1942), “El Aleph” (1945) o en “El Libro de arena" (1975). La definición del concepto rizoma por Borges en "El jardín de senderos que se bifurcan" (1941) reza:

En todas las ficciones, cada vez que un hombre se enfrenta con diversas alternativas, opta por una y elimina las otras; en la del casi inextricable Ts'ui Pên opta -simultáneamente-por todas. Crea, así, diversos porvenires, diversos tiempos, que también proliferan y se bifurcan. En la obra de Ts'ui Pên todos los desenlaces ocurren; cada uno es el punto de partida de otras bifurcaciones. $[\ldots]$

Creía en infinitas series de tiempos, en una red creciente y vertiginosa de tiempos divergentes, convergentes y paralelos. Esa trama de tiempos que se aproximan, bifurcan, cortan o que secularmente se ignoran, abarca todas las posibilidades. (OC 1989, I, 478-479)

Deleuze y Guattari lo formulan así:

Un rhizome ne cesserait de connecter des chaînons sémiotiques, des organisations de pouvoir, des occurrences renvoyant aux arts, aux sciences, aux luttes sociales. Un chaînon sémiotique est comme un tubercule agglomérant des actes très divers, linguistiques, mais aussi perceptifs, mimiques, gestuels, cogitatifs: il n'y a pas de langue en soi, ni d'universalité du langage, mais un concours de dialectes, de patois, d'argots, de langages spéciales. Il n'y a pas de locuteur-auditeur idéal, pas plus que de communauté linguistique homogène. (1976: 20) 


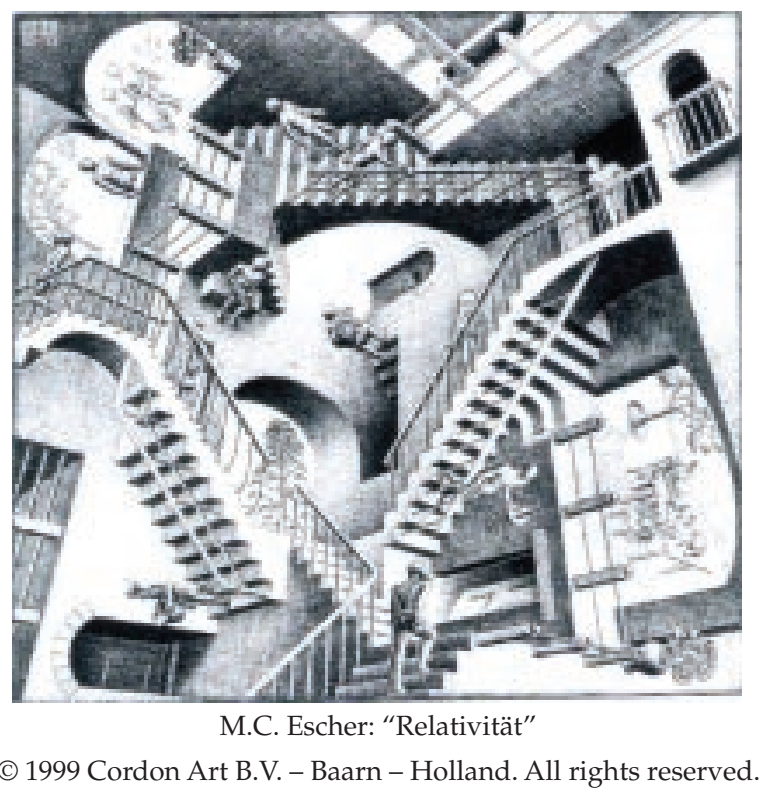

Cuando Borges en el prólogo a "Ficciones" dice que "[m]ejor procedimiento es simular que esos libros ya existen y ofrecer un resumen, un comentario" (1972/21989, 429) y continúa con que ha "preferido la escritura de notas sobre libros imaginarios" (ibíd.) (las itálicas son mías), cualquier lector se pregunta qué significa 'simular' y escribir "notas sobre libros imaginarios", ya que lógicamente es una paradoja: el querer escribir sobre algo que no existe y cuál es la necesidad para proceder de esta forma. ¿Cómo podemos resolver la paradoja? Propongo una posibilidad. Borges replantea y reformula primero que nada un problema antiguo: ya no se trata de la oposición 'realidad vs. ficción' (mimesis de la realidad), ni de las referencias textuales (intertextualidad o mimesis de la literatura, mimesis de la ficción), sino de un acto virtual, de una literatura sin referencia, sin precedentes, por esto, el término 'simulación' adquiere una significación que no es la mimética y cotidiana que todos conocemos, sino aquella que Baudrillard acuña en 1981: "Le simulacre n'est jamais ce qui cache la vérité -c'est la verité que cache qu'il n'y en a pas. Le simulacre est vrai" (ibíd., 10), con lo cual Borges da una nueva y revolucionaria definición al término de ficción y de literatura.

\section{Conclusión:}

Borges formula en los años cuarenta pensamientos centrales de tres filósofos fundamentales de Occidente: los del rizoma, de la simulación, de la deconstrucción y diseminación de la significación.

\section{2 "Teoría de Mundos Diversos": Borges y Everett III}

Los textos de Borges no solamente anticipan las ideas expuesta más arriba, sino también la teoría postcuántica de la Many World Theory y de la construcción del Web.

“El Aleph" (1943), “El jardín de senderos que se bifurcan” (1941) y “Tlön, 
Uqbar, Orbis Tertius" (1940), representan las primeras ideas de la "Many Worlds Theory" de Hugh Everett III que él formula entre 1955-1957 en Princeton y, además, del sistema Web y del Hipertexto, lo que luego es confirmado en "El libro de arena" (1975). El término que aparece allí, el de hipervolumen, habla por sí mismo. Es precisamente en la trasgresión de las disciplinas y en la visión de mundos en otros campos fuera de la literatura donde radica la fascinación de los relatos de Borges.

La concepción de mundos de Borges está estrechamente relacionada con la "Many Worlds Theory" en la que se parte del presupuesto de la existencia de muchos mundos paralelos como consecuencia de interpretaciones alternativas de procesos mecánicos cuánticos de medida. Se postula que con cada medida de una unidad de medida mecánico cuántica (Observablen) tiene lugar una fisión (división) de todas las posibilidades de realización de la unidad de medida, esto es, en diversos mundos. En el contexto del formalismo de la cosmología cuántica, esta interpretación promete la existencia de universos paralelos (véase Andreas Müller [http://www.lsw.uniheidelberg.de/users/amueller/lexdt_v. html], Astrofísico de la Universidad Heidelberg). Este presupuesto no ha sido confirmado hasta la fecha en forma directa o indirecta, aunque en los últimos años ha cobrado una gran actualidad en la persona de David Deutsch.

Everett III, el gran alumno de Wheeler, otro grande de la física conocido en otras cosas por la "ecuación Wheeler-Dewitt", propuso en su tiempo una nueva y revolucionaria interpretación de la mecánica cuántica "that denies the existence of a separate classical realm and asserts that it makes sense to talk about a state vector for the whole universe" (DeWitt/Graham 1973), que consiste en la idea de mundos diversos como DeWitt y Graham explican en el prólogo a The Many-Worlds Interpretation of Quantum Mechanics.

No es un mero azar que los autores que editan el trabajo de Everett III, de John Archibald Wheeler, Bryce Seligman DeWitt, Leon Neil Cooper (premio Nobel de Física de 1972), D. van Vechten y Robert Graham comiencen con dos citas-lemas, una de "El jardín de senderos que se bifurcan" de Borges (vid. la cita ya mencionada más arriba, vid. aquí DeWitt/Graham 1973, 5), y la segunda de William James (uno de los filósofos favoritos de Borges): "Actualities seem to float in a wider sea of possibilities from out of which they were chosen; and somewhere, indeterminism says, such possibilities exist, and form part of the truth".

Los físicos descubren y formulan aquello que escritores y filósofos habían pensado y formulado, pero no son críticos literarios, ni los expertos de la literatura de Borges los que hacen un aporte semejante. Este hecho revela la necesidad inminente de la transdisciplinariedad.

Ambos, Borges a partir de 1939 y Everett III a partir de 1955, se ocupan de la idea de un "continual splitting of the universe into a multitude of mutually unobservable but yielded a definite result and in most of which the familiar statistical quantum laws hold" (ibíd.).

He aquí un conocido ejemplo de la mecánica cuántica, el del gato de Schrödinger, ejemplo que, como veremos, podría venir de Borges: 
Podríamos decir que el gato de Schrödinger en nuestro mundo está muerto o vivo y que en otra realidad ha sucedido exactamente lo contrario. En los viajes temporales partimos de la base que si, por ejemplo, una persona mata a su padre antes de su nacimiento, ésta misma desaparece. Si existiesen varias realidades, sería posible que la persona solamente en una realidad mate a su padre y ella misma siga en otra, porque existen varias realidades. Pero no existe la seguridad que la teoría sea verdadera. (Lukas Czarnecki http:/ / www.hpwt.de/Quanten2.htm)

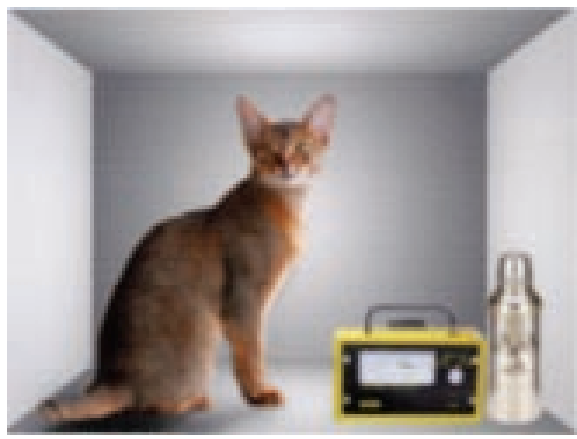

(C) Wikipedia

http://de.wikipedia.org/wiki/Schr\%C3\%B6dingers_Katze

La transmisión de las funciones de ondas mecánico-cuánticas nos conduce hasta el momento de abrir el cajón a un gato que a la vez está muerto y vivo. Y en Borges la fórmula reza:

Alguna vez, los senderos de ese laberinto convergen: por ejemplo, usted llega a esta casa, pero en uno de los pasados posibles usted es mi enemigo, en otro mi amigo.

El ejemplo del "gato de Schrödinger", según el cual éste puede estar vivo o muerto dependiendo de la realidad en la que se encuentre corresponde exactamente al enunciado de Borges de que un personaje puede ser en un mundo presente su enemigo, en otro su amigo.

Borges nos propone no limitar la realidad, porque no siempre podemos captarla en todas su facetas y posibilidades, sino que debemos presuponer que otras muchas y diversas son pensables (de allí que el pensamiento de Borges se sitúe al límite de lo pensable en el contexto de postulados normativos de la realidad).

Sobre la base de la teoría cuántica, Everett III concluye que el universo no está reducido a la realidad que vemos de tal modo que otros possible worlds se ramifican o bifurcan y por esto existen muchos otros possible worlds o bifurcaciones temporales más de los que presuponemos. El concepto de 'onda' implica diversos estados de tal forma que mientras un estado en un mundo desaparece, en otro continúa existiendo.

Y, ¿cómo formula Borges este fenómeno? La cita que opera como definición del rizoma (vid. más arriba) es la que define la teoría de los muchos mundos: 
Crea, así, diversos porvenires, diversos tiempos, que también proliferan y se bifurcan. [...] todos los desenlaces ocurren; cada uno es el punto de partida de otras bifurcaciones. Alguna vez, los senderos de ese laberinto convergen: por ejemplo, usted llega a esta casa, pero en uno de los pasados posibles usted es mi enemigo, en otro mi amigo.

$[\ldots]$

Creía en infinitas series de tiempos, en una red creciente y vertiginosa de tiempos divergentes, convergentes y paralelos. Esa trama de tiempos que se aproximan, se bifurcan, se cortan o que secularmente se ignoran, abarca todas las posibilidades. (Borges 1972/21989, I, 478-479)

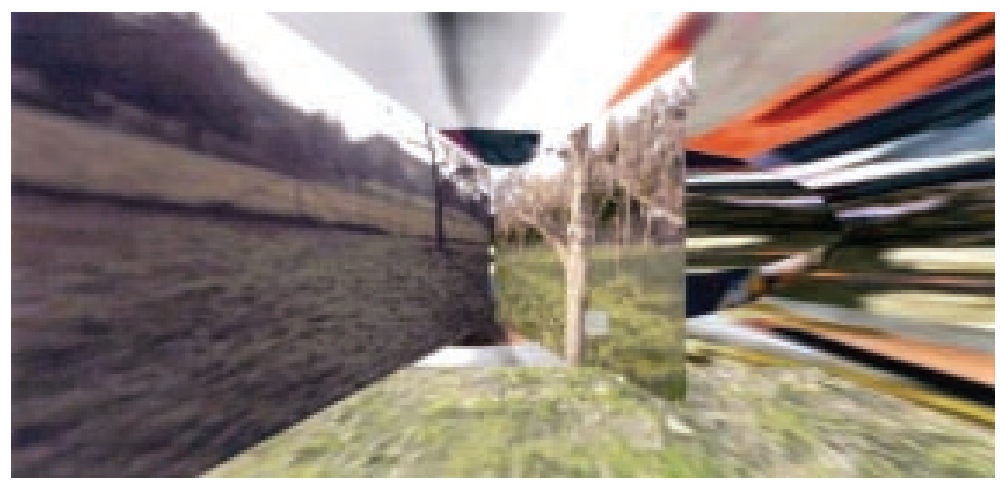

(C) Jürgen Meier (architektur\&medien Leipzig): “El jardín de senderos que se bifurcan”.

Los enunciados "diversos porvenires", "diversos tiempos", "proliferar", "bifurcarse", "pasados posibles", "infinitas series de tiempos" (= ondas o mundos paralelos), "red creciente y vertiginosa de tiempos divergentes, convergentes y paralelos" y "trama de tiempos" indican con toda claridad que Borges conocía tanto la teoría de la relatividad especial (Einstein 1905) como la general (Einstein 1915) de donde Borges formulará la teoría de los mundos paralelos a su manera, y de donde Everett III, en la mitad de los años cincuenta, la formulará para la física. Las concepciones de sistemas que se mueven relativamente lineal y uniformemente o aquella idea usual de lo absolutamente simultáneo fueron desplazadas por la concepción de la teoría de la relatividad especial y por aquella de Borges de la relatividad de lo simultáneo. Dos acontecimientos se encuentran, por ejemplo, espacialmente separados, pero al mismo tiempo se encuentran en un sistema inercial, se bifurcan temporalmente en un sistema en movimiento. Borges comparte también la idea de la influencia recíproca de acontecimientos separados espacial y temporalmente, esto es, que "cada proceso físico puede influir en otro a través de la mediación de un efecto que se propaga con una velocidad de la luz" (Brockhaus Multimedial 2005). Con la teoría de la relatividad general, Borges comparte la idea de un mundo espacio-temporal tetradimensional como así también la existencia de campos de gravitación o la cambiante "curvatura o encorvadura del espacio" de un punto a otro (Krümmung des Raumes). Un acontecimiento existe por esta razón en dos mundos y estados al mismo tiempo. 


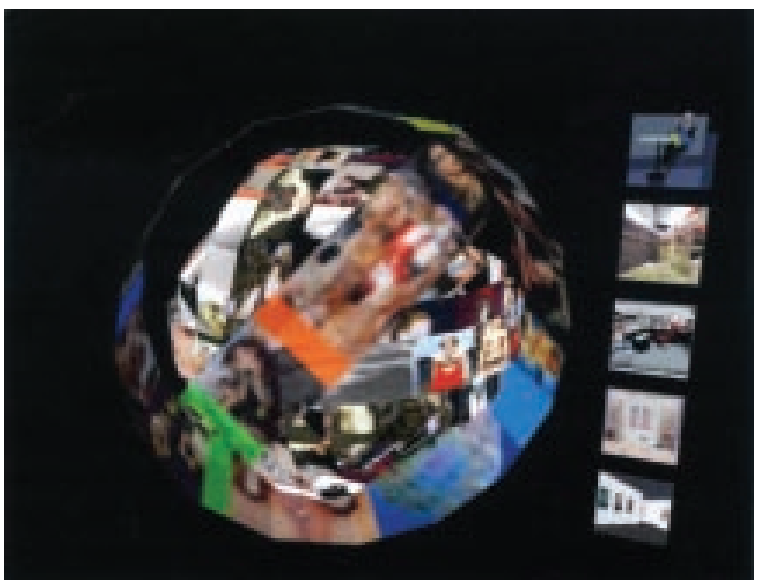

(C) Jürgen Meier (architektur\&medien Leipzig): “El Aleph”.

\section{Como se ilustra en "El Aleph"}

Arribo ahora, al inefable centro de mi relato; empieza, aquí mi desesperación de escritor. Todo lenguaje es un alfabeto de símbolos cuyo ejercicio presupone un pasado que los interlocutores comparten; ¿cómo transmitir a los otros el infinito Aleph que mi memoria apenas abarca? [...] Quizás los dioses no me negarían el hallazgo de una imagen equivalente, pero este informe quedaría contaminado de literatura, de falsedad. Pero lo demás, el problema central es irresoluble: la enumeración, siquiera parcial, de un conjunto infinito. En ese instante gigantesco, he visto millones de actos deleitables o atroces; ninguno me asombró como el hecho de que todos ocuparan el mismo punto, sin superposición y sin transparencia. Lo que vieron mis ojos fue simultáneo: lo que transcribiré, sucesivo, porque el lenguaje lo es.

En la parte inferior del escalón, hacia la derecha, vi una pequeña esfera tornasolada, de casi intolerable fulgor. Al principio la creí giratoria; luego comprendí que ese movimiento era una ilusión producida por los vertiginosos espectáculos que encerraba. El diámetro del Aleph sería de dos o tres centímetros, pero el espacio cósmico estaba ahí, sin disminución de tamaño, Cada cosa [...] eran infinitas cosas, porque yo claramente las veía desde todos los puntos del universo. ("El Aleph" en OC 1972/21989, I, 625)

\subsection{Borges y los 'New Media' (mundos virtuales-digitales)}

El pensamiento y la práctica literaria de Borges son una gigantesca red de citas de unidades, fragmentos, elementos, que se dispersan y desplazan rizomáticamente, que se amplían y bifurcan construyendo así diversos mundos. Esta red implosionante tiene, en el caso de Borges, su punto de partida arbitrario y virtual en un LIBRO que es la suma de fragmentos de muchísimos otros libros y que a su vez nos llevan a una 
infinidad de otros tantos etc., continuamente trasgrediendo los límites de la disciplina. En un libro se refleja la suma de la totalidad de los libros, el libro total o la biblioteca que a su vez nos remite a infinitas bibliotecas. Así tenemos una enmadejada red siempre oscilante, nómada, en permanente ampliación, retorno fragmentario, parcial y variado de textos y su desaparición.

Por esto, los procedimientos de Borges para la construcción de textos equivalen en un micronivel (del texto individual) a la construcción de la biblioteca. Borges crea un mundo de libros e ideas atópicas no perceptibles que a su vez equivalen estructuralmente al hypertext, al hyperlink y así al Web. Tenemos una equivalencia u homología entre las construcciones literarias de Borges y de sus "infinitos laberintos" sin centro y con una infinidad de entradas y salidas, un regressus ad infinitum que en un macronivel corresponden al sistema de multiuniversos y de Internet.

Por virtual entiendo en Borges no solamente el concepto medial de Baudrillard en el sentido del reflejo pre-supuesto o simulado de una inexistente realidad, esto es, de una hiperrealidad, sino además el concepto físico y filosófico en el sentido de que la capacidad del ser humano de entender es infinita y en este contexto la virtualidad es "una cualidad central y fundamental del multiuniverso", como Deutsch (1996, 118) lo concibe.

Mi interpretación de la literatura y del pensamiento de Borges ha sido confirmada en forma ejemplar no tan sólo por la mención de Borges en obras de filosofía y física fundamentales -como he venido exponiendo-, sino también por el lugar fundamental que Borges goza en los New Media como parte de las ciencias mediales, por ejemplo, en The New Media Reader de MIT Press, compendio editado por Noa Wardrip-Fruin y Nick Montfort (2003) y concebido por Michael Crumpton. Este Reader, o mejor dicho, la historia apasionante de los New Media, es inaugurada con el relato de Borges, "El jardín de senderos que se bifurcan" editada definitivamente en 1940, año del comienzo de los conceptos mediales, según los autores. Además se recomiendan las lecturas de "La biblioteca de Babel" y de "El libro de arena", obras que han constituido hace años la base de toda mi teoría del pensamiento y obra de Borges. También se recomienda Rayuela de Cortázar. Otros autores que forman parte del "new media turn" son Vannevar Bush, Alan Turing, Norbert Wiener, William S. Burroughs, y dentro de los "oulipistas" se incluyen Raymond Queneau, Claude Berge e Italo Calvino, como así también un representativo número de ensayos de filósofos (como Baudrillard, Deleuze) y teóricos de los New Media (como Nelson).

La cita del "Aleph" dada más arriba es a la vez el ejemplo más perfecto del Web. La 'desesperación' de la que Borges habla consiste en la incapacidad de la escritura, a raíz de su inevitable linealidad, de su taxonomía causal, normativa y jerárquica de representar la infinitud del mundo, o dicho de otra forma, de describir lo absoluto y la infinita diversidad de lo simultáneo, de la escenificación y de la visualización de lo observado y descrito donde todo sucede al mismo tiempo y en un mismo punto. La creación de la absoluta virtualidad.

Otro pasaje que nos lleva al mundo Web es este pasaje inicial en el "Libro de arena": 
La línea consta de un número infinito de puntos; el plano, de un número infinito de líneas; el volumen, de un número infinito de planos; el hipervolumen, de un número infinito de volúmenes... (Borges, OC 1972/2²1989, II, 95)

Murray (2003) ve en Borges un autor que en el contexto de las visiones de los nuevos medios estableció estándares y bases fundamentales, ya que Borges estaba muy consciente del fracaso e incapacidad de la escritura en su mediatizante linealidad para la percepción y descripción de un mundo de ideas y de fenómenos complejos. Murray subraya que Borges fue el primero en situarse en un mundo cultural global y que estaba fascinado por "flutter of meaning across cultural boundaries" (ibíd., 3), esto es, por la diseminación de la significación más allá de fronteras culturales. Al mismo tiempo es importante el bagaje de conocimiento que Borges tiene sobre diversas disciplinas. La literatura es para él un mero medio -naturalmente que uno muy querido- para transportar diversos mundos y juegos de ideas cambiando continuamente de perspectiva, como afirma Murray.

Son perspectivas renovadoras, nómadas en un infinito proceso de translación, como tematización de las infinitas posibilidades de elección, selección, decisión y proliferación, procesos que Borges denomina "pululación", término tomado por Murray y transportado al inglés como "pullulating". Se trata de un proceso de intersecciones. Como ejemplo dentro del contexto de los New Media es adecuado "El jardín de senderos que se bifurcan" que nos hace consciente de las numerosas posibilidades mediales y de diversos mundos, ésta es una obra "that has the shape of a labyrinth that folds back upon itself in infinite regression", una "dizzying vision", y que caracteriza al siglo XX (ibíd.). A pesar de que Borges parte de un concepto del libro y de la biblioteca, él trasgrede esta estructura rellenando un libro (relato) por medio de la citación de numerosísimos otros libros. A través de esta permanente implosión, Borges trasciende la categoría 'libro' a un campo de una red proliferante de significantes constituyendo un hipertexto y entrando así en diversos mundos.

Carece de importancia que Borges naturalmente no supiera nada de ordenadores ni de Internet, que en ese entonces no existían. Importante es solamente la estructura de su pensamiento y de su práctica literaria, que el libro sea para él una superficie con infinitos nudos y líneas, una red de madejas en permanente expansión, como la Web, que su proceder literario corresponda a la estructura y procedimiento de la Web y a la práctica artística del user; eso es lo único importante: la homología estructural. Consideramos de gran importancia que su pensamiento anticipe y anuncie una nueva forma de pensar, una nueva forma de ciencia y de pensar mundos que finalicen con un tipo de metafísica humanística-dualista, tarea comenzada ya por Nietzsche y seguida por Heidegger y los filósofos postmodernos. Borges, naturalmente, rechazaría esta aseveración, quizás con enojo, pero eso no nos lleva adelante, ya que sus propios textos -"El Aleph", "El jardín de senderos que se bifurcan", “Tlön, Uqbar, Orbis Tertius" o "El libro de arena"- se sitúan más allá de la metafísica dualista, más allá de la literatura encarcelada en la tríada semiótica.

La literatura de Borges -y aquella de algunos de sus contemporáneos y de sus sucesores- consiste en la escenificación de lo que van a ser la ciencia y los sistemas 
mediáticos del siglo XX: "[...] tuning up our sense of existential befuddlement before the scientifically revealed world of the twentieth century", como indica Murray $(2003,4)$.

Ya he referido cómo Borges anticipa y formula en su totalidad teorías centrales de la postmodernidad y postcolonialidad, del rizoma, de la simulación y la diseminación que sólo decenios más tarde serán formuladas en la filosofía postmoderna y en la física. Borges es una permanente referencia para el desarrollo de estas epistemologías. Mi interés ha radicado en mostrar la dirección que toma el pensamiento de Borges y no en concentrarme en el objeto -que es indiferente-, por esto, sólo importa la analogía y homología estructural y sistémica. Ambos, Borges y los New Media, comparten la simulación de mundos virtuales. Mientras Borges tan solo logra la descripción de un "mundo de tiempo real" a raíz de la linealidad de la escritura -como indicaba más arriba-, los expertos de los New Media consiguen producir mundos virtuales en tiempo real. El pensamiento y la práctica literaria de Borges comparten el procedimiento enciclopédico con los New Media, especialmente con el ordenador, con Internet y con su adjunto, el hipertexto, esto es, cuatro procedimientos básicos y fundamentales del ordenador: el procedimiento 'procedual', 'participante', 'espacial' y el 'enciclopédico' y con ello el intento de representar el universo y el conocimiento total en su total complejidad condensado en un punto, que es lo que alegóricamente representan “El Aleph", "El libro de arena”, “El jardín de senderos que se bifurcan".

La inteligente concepción del libro de los autores de los New Media confirma mi interpretación sobre la afinidad estructural entre Borges (y otros autores) con el desarrollo de los New Media:

The difference is not so much in what they describe as in their orientation to it. The humanists see the contradictions and limitations of the great systems of thought and it causes them to question the very project of systemized thinking [...] (ibíd.: 4),

que apunta a la importancia de ver "more closely at the rich interplay of cultural practice and technical innovation" (ibíd.).

Borges construyó un 'metalibro' en el cual están contenidos todos los libros, todos los signos, todas las bibliotecas y todas las disciplinas y esto simultáneamente en ese metalibro que es "El Aleph" o "El libro de arena" donde podemos navegar infinitamente. Términos como "flickering focuses", "slippin way", "flutter of meaning" o "proliferation" y "pullulation of possibilities and path", remiten a la división entre significante y significado y representan las bases terminológicas y procesuales de lo que hoy entendemos por virtualidad y multiplicidad de mundos. Lo que Borges piensa y formula en los años cuarenta va a ser "[...] the intellectual predicament of the second half of the twentieth century", como Murray $(2003,4)$ indica.

Lo que Foucault (1966) dice con respecto a Cervantes, que el artista como el bufón o el gracioso tienen una particular antena para las profundas transformaciones (especialmente en el caso de cambios de paradigmas) de tal forma que éstos son los primeros en detectar y evocar el mundo nuevo que se anuncia (en el paso de la Edad Media al Renacimiento se trataba de la percepción del término de las analogías y el 
comienzo de la diferencia), es válido en forma muy particular para Borges y para el siglo XX y el comienzo del XXI. Podemos afirmar: Borges infinito, Borges virtual razón por la cual denominé en Leipzig un coloquio dedicado a Borges en 1999 y su respectivo volumen "El siglo de Borges". Como vemos, este título no era solamente justificado, sólo que en ese entonces no mencionábamos el siglo XXI. Borges, y otros autores, crearon diversos mundos a través de la alegoría, equivalencia y homología, es decir, diseños para concepciones y construcciones científicas y mediales:

The engineers draw upon cultural metaphors and analogies to express the magnitude of the change, the shape of the as yet unseen medium. The storytellers and theorists build imaginary landscapes of information, writing stories and essays that later become blueprints for actual systems.

Gradually, the braided collaboration gives rise to an emergent form, a new medium of human expression. (Murray 2003, 5)

Lo que he descrito con el concepto de diseminación y de rizoma, término que también juega un papel importante en el volumen New Media con el ensayo correspondiente de Deleuze, se desarrolla paralelamente al concepto de hipertexto. Murray apunta que Borges es la fuente del término de rizoma (como ya lo había expuesto yo en 1992, basándome en el mismo texto, en "El jardín de senderos que se bifurcan"):

The two philosophers suggested a new model of textual organization to replace the ideologically suspect hierarchies of the old print-based world. [...] It was as if Deleuze and Guattari had dug beneath the forking path garden of Borges [...] and come up with an even more profound labyrinth, but one that offers the hope of knowability and a metaphor of healthy growth. The potato root system has no beginning no end, and grows outward and inward at the same time. It forms a pattern familiar to computer scientists: a network with discrete interconnected nodes. Here was a way out of the pullulating paralysis, one that beyond the subversion of all existing hierarchies. Here was a way of constructing something new. The humanist project of shredding culture had found a radical new pattern of meaning, a root system that offered a metaphor of growth and connection rather than rot and disassembly. (Murray 2003, 9)

Con lo expuesto espero haber dejado en claro que Borges con su literatura trasciende la literatura de tal forma que lo importante no es la fuente de las referencias concretas, sino los mundos y posibilidades que Borges abrió con su pensamiento.

Y muy en este sentido, Borges supera (va más allá de) aquello que él admiraba en Kafka: que él, Borges, no sólo produce también una literatura que trasciende su lugar de escritura o el lugar de la anécdota, sino que trasciende la literatura como medio y sus propias finalidades con un alcance que seguramente a él mismo le fue desconocido. Borges se superó a sí mismo y por ello se transforma en una figura de los mundos virtuales y múltiples (Denkfigur virtueller und vieler Welten).

Estas visiones mediáticas de Borges son formuladas en los años sesenta cuando se produce la primera onda de científicos de ordenadores, cuando la concepción de 
Internet se anuncia con Weizenbaum o Nelson y se introduce el término de hypertext; en este momento del desarrollo medial-tecnológico se encuentra en el centro del interés el concepto procedimental-partícipe-espacial de la enciclopedia.

These can be fictional landscapes, like Borges's labyrinthine garden, or they can be information spaces, like Bush's memex machine. The sense of following a trail is the same in both cases, and it is a sense that creates the pleasurable experience of immersion, of moving within a capacious, consistent, enveloping digital environment rather than just looking at it. (Murray 2003, 6)

Borges es el primero en formular el concepto de rizoma y de hipertexto. Aun cuando él no emplee estos términos, no olvidemos su concepto de "hipervolumen" en "El libro de arena" en el sentido de superposiciones ajerárquicas de infinitos niveles textuales, un concepto que se anticipa al de Nelson:

Let me introduce the word "Hypertext" to mean a body of written or pictorial material interconnected in such a complex way that it could not conveniently be represented or represented on paper. It may contain summaries, or maps of its contents and their interrelations; it may contain annotations, additions and footnotes from scholars who have examined it. [...] Such a system could grow indefinitely, gradually including more and more of the world's written knowledge. However, its internal file structure would have to be built to accept growth, change and complex informational arrangements.

Films, sound recordings, and video recordings are also linear strings, basically for mechanical reasons. But these, too, can now be arranged as non-linear systems [...] The hyperfilm -browsable or variasequenced movie-is only one of the possible hypermedia that require our attention. [...] The criterion for this prefix is the inability of these objects to be comprised sensibly into linear media, like the text string, or even media of somewhat higher complexity. (Nelson 1965, 144)

El concepto de hipertexto y rizoma forman una unidad en Borges, ya que tanto para Deleuze como para Nelson se trata de un sistema antiespacial y atemporal donde se encuentran diversos sistemas simultáneamente: "Thus may help integrate, for human understanding, bodies of material so diversely connected that they could not be untangled by the unaided mind" (Nelson 1965, 144).

Un tercer ejemplo. Cuando Borges en El tamaño de mi esperanza habla del criollismo lo hace fuera de las normas establecidas, no entiende ni un término nacionalista-progresista (con la mirada hacia Europa) ni una forma nacionalistaesencialista local. Lo entiende como "conversador del mundo" (Borges 1926/1994, 14), para luego años más tarde en "El escritor argentino y la tradición" afirmar que:

[...] nuestro patrimonio es el universo; ensayar todos los temas, y no podemos concretarnos a lo argentino para ser argentinos: porque o ser argentino es una fatalidad y en ese caso lo seremos de cualquier modo, o ser argentino es una mera afectación, una máscara [...] [y] [c]reo que si nos abandonamos a ese 
sueño voluntario que se llama la creación artística, seremos argentinos y seremos, también, buenos o tolerables escritores. (OC I, 1972/21989, 274)

Borges está allí tratando no solamente un fenómeno literario, sino un problema de la teoría de la cultura.

Lo que Borges desde un comienzo está haciendo (e incluyo aquí también Inquisiciones de 1925 y El idioma de los argentinos de 1928, Evaristo Carriego de 1930 e Historia de la infamia de 1935) es redefinir un territorio cultural desde su enunciación que se encuentra en las orillas en sentido menos geográfico, sino altamente semiótico y epistemológico; se encuentra entremedio, en una simultaneidad del aquí y del allá, de la que Bhabha habla en The Location of Culture en términos de "in-between", "mimikry", "unhomely". Borges se está ubicando con lo que respecta a la tradición literaria y cultural argentina y europea en un espacio intermedio de permanente negociación de identidades culturales y está rompiendo con todo tipo de normas de valorización estética (canónica) de obras e inicia con esto un debate que luego se va a conocer como postmodernidad (bajo la fórmula de "double codification" Fiedler) y como postcolonialidad ('contra-escritura-re-escribir' / 'Wider-/ Wiederschreiben'). Para poder comprender la dimensión en que Borges se movía, no basta reconstruir tan sólo su contexto local inmediato o su contexto literario internacional, sino que es necesario ubicarlo dentro de un planteamiento teórico actual que requiere otra competencia que la de la CL.

Con los ejemplos anteriores he querido tan solo mostrar las relaciones entre la escritura y el pensamiento de Borges, la física y los New Media, sin querer disminuir otras posibles y muy válidas interpretaciones. He querido más bien mostrar otra vertiente hasta la fecha algo descuidada. El problema de la ficción como algo literario pasa a ser en Borges un problema de la construcción de diversos sistemas y, en ese momento, nos enfrentamos a un problema matemático, físico y filosófico o lingüístico. La paradoja, apuntada más arriba, la podemos interpretar en este caso recurriendo a parámetros filosóficos dentro de la filosofía postmoderna y, sin el contexto epistemológico postmoderno, no podríamos entender el contexto de argumentación de Baudrillard, Foucault o Deleuze, ni muchos menos ayudarnos a resolver esta paradoja borgeana.

Para llegar a semejantes conclusiones -que no puedo profundizar en este lugar, pero que las he tratado en diversas publicaciones- he tenido que traspasar los límites tradicionales de la CL y entrar en un campo que no es el de nuestra tarea cotidiana, para así descubrir una serie de factores fundamentales no sólo en la obra de Borges, sino del pensamiento del siglo XX. Naturalmente que se podría objetar que primero se hubiesen podido analizar estas obras bajo un punto de vista estrictamente literario sin recurrir a textos filosóficos. Por el contrario, argumentaría, que la CL no ha interpretado satisfactoriamente las paradojas borgeanas; segundo, se podría criticar que solamente a raíz de la lectura filosófica se le ha imputado a Borges algo que le es extraño, esto es, una interpretación arbitraria y hegemónica que privilegia un pensamiento europeo occidental, es decir, una interpretación hegemónica que descontextualiza la obra de Borges. Podría ser, no obstante este tipo de reservas son válidas para todo tipo de aproximación interpretativa. 
Ahora bien, ¿cómo se decide la verdad de una interpretación, para no caer en un relativismo desenfrenado? A mi parecer existe solamente una solución, una inmediata y otra a largo plazo: dentro de una ciencia transversal no existe la verdad, sino una verosimilitud científica, es decir, la capacidad de convencer a otro sujeto de lo propuesto, y si no de inmediato, en un proceso de debate.

El problema de la ficción como algo literario pasa a ser en Borges un problema de sistemas de construcción teórico-científico-cultural-epistemólgico-sígnico, en ese momento, nos enfrentamos a un problema matemático, físico y filosófico. La paradoja, en este caso, la podemos interpretar recurriendo a parámetros filosóficos dentro de la filosofía postmoderna y, sin el contexto epistemológico postmoderno, no podríamos entender el contexto de argumentación de Baudrillard, Foucault o Deleuze, ni muchos menos ayudarnos a resolver esta paradoja borgeana.

\subsection{Cervantes o el detector de la Época Moderna}

Otra obra tratada por Foucault en Les mots et les choses es el Quijote de Cervantes, autor quien, según el filósofo francés, es el primero en detectar el cambio de paradigma de la Edad Media a la Época Moderna, donde se dectata el tránsito de un sistema de pensamiento o de signos a otro que implica una crisis también de la representación que revela cómo un sistema antiguo y ya agotado se va superando y al mismo tiempo va anunciándose el nuevo. Como Cervantes en el Quijote, el libro de Foucault trata el fenómeno semiótico-epistemológico de las similitudes y diferencias que se revelan como epistemas-bases de las épocas analizadas por él, como resultados de rupturas epistemológicas y de crisis sígnicas. Su trabajo describe cómo Cervantes lee e interpreta el mundo y los signos. Así, el Quijote no es tan sólo una obra paródica-cómica que representa un cambio de paradigma en la tradición de la narración como lo explicaba el formalista ruso Sklovsky en los años veinte del siglo pasado, sino que aprendemos que Don Quijote/Cervantes emprenden un viaje en busca de los signos perdidos, que lo llenan de nostalgia, pero de donde surgirán los signos de la Época Moderna. Don Quijote/Cervantes emprenden un camino, abandonan la patria conocida para arrojarse a lo nuevo, a lo incierto: Don Quijote emprende la tarea de restituir el mundo del mito, de los caballeros armados, de los escudos de armas, de los símbolos y de las unidades y analogías que contienen al mundo entero encauzando su trayectoria en un viaje de aventuras determinado por la mimesis. Ese viaje es el motivo del autor implícito/Cervantes para declarar como superado el antiguo mundo, la antigua organización de los signos, la transformación de las analogías en similitudes y diferencias.

Cervantes se encuentra bajo la influencia de una época caracterizada por una fragmentación y disociación del pensar que se manifiesta en la escisión del signo y de lo designado, de la superficie y la profundidad. Las similitudes ya no hallan signaturas icónicas que les correspondan, se convierten en no-similitudes y la interpretación falla porque sobreviene una profunda inversión de las relaciones sígnicas. Los objetos de la superficie son tomados por algo distinto de lo que son en la profundidad; superficie y profundidad ya no se encuentran en correspondencia. 
Ahora domina el irracionalismo de donde proceden los fetiches, las quimeras, las creaciones de la fantasía, las alegorías, las metáforas y las confusiones (quid pro quo) que han contribuido a dar el sello característico a todo el sistema cultural del Barroco. El contenido del lenguaje se contrae hasta quedarse en un simple armazón retórico, los personajes actuantes, por ejemplo Don Quijote, son el resultado de mera retórica, el producto de libros (el mismo Quijote es un libro sobre libros), un grafismo, una letra. El lenguaje ya no está en condiciones de equiparar similitudes con naturalezas, quien busca ha de encontrar ahora pruebas de las nuevas relaciones entre similitudes y naturaleza. El establecimiento de tal relación no le resulta posible porque el lenguaje está todavía preso en el orden antiguo: los molinos de viento se convierten en gigantes épicos, los rebaños de ovejas en ejércitos. Don Quijote tiene que probar lo que decían los libros y lo que ya no tiene validez. Pero al no existir ya analogías ni unidades, las formulaciones textuales se prueban mediante su inversión, los signos se ponen de cabeza. Los actos engañosos, disparatados son la única demostración que queda.

Aunque los libros no contienen la verdad, se los imita servilmente. Impulsados por la fantasía, los personajes que pueblan la literatura barroca intentarán establecer similitudes mediante la astucia y la ilusión de los sentidos. El lenguaje se vuelve mera visión, delirio. Las signaturas producen irritación como consecuencia de la desemiotización que se da, no destruyen el sistema cultural sino que lo deconstruyen. Con la transición de un tipo de organización semántico-simbólico-cultural a otro sintáctico-analítico-ordenador, donde el empirismo y los intereses prácticos y reales están en primer plano, tiene lugar una transformación semiótica que llevará a una organización nueva de signos y de pensar. El nuevo tipo de organización sintácticocultural de los signos en tiempos de crisis histórico-epistemológica -cuando las instituciones, los postulados, los valores, las teorías y los ideologemas sociales son desautorizados- asume la función de transemiotizar modelos del mundo.

En el transcurso del siglo XVII la similitud y sus signaturas análogas ya no representan la forma válida del saber, sino que ofrecen ocasión de error. Por todas partes se destacan los embustes de la similitud, pero ahora se sabe que son fantasías. Fue -como anota Foucault-la época privilegiada del trompe-l'ail, de la ilusión cómica, del teatro, del quid pro quo, de los sueños y de las visiones. Fue la época de las ilusiones de los sentidos y de los juicios erróneos, la época en que las metáforas, las comparaciones y las alegorías determinaron el modelo semiótico-cultural.

El acontecimiento radica en Cervantes, al fin de su viaje como búsqueda de una nueva redistribución y determinación de la unidad perdida entre significado, significante y referente: el acontecimiento representa una restitución como lectura del mundo a espaldas de la literatura o la lectura de la literatura a espaldas del mundo. En Borges se encuentra el acontecimiento en la búsqueda de la absoluta percepción sin espacio ni tiempo; ya no se trata de la lectura del mundo, sino de la codificación sígnica de la percepción como mundo.

El creador poético-literario, esto es la literatura, se encuentra en una situación privilegiada: él es quien hace que una crisis, que lo nuevo, lo tabuizado salga a la superficie y sea debatido. La literatura es el único lugar de la individualidad y donde 
la diferencia del individuo y su identidad encuentran un lugar adecuado de tratamiento. El creador, inconsciente o conscientemente, sin quererlo o queriéndolo, manifiesta la crisis: la literatura irrumpe, nace de la crisis, del conflicto, de lo conflictivo. La literatura anticipa lo porvenir y analiza con la perfección de un bisturí la realidad contemporánea, la literatura articula las tensiones entre el yo y el mundo, entre norma y deseo, pone a disposición un diagnóstico representado como ficción, que no es otra cosa más que grandes y duras verdades, la literatura es el refugio y el medio más íntimo que tiene el sujeto.

¿Y cuál es la relación entre literatura, y podríamos decir arte o teatro, las Humanidades y las Ciencias Sociales, con sus diversas teorías al servicio a la interpretación? Existe el prejuicio que se establece hasta más tardar en la Ilustración europea, en su ideología del progreso, que la literatura o el arte y con ello las ciencias que los acompañan no obedecen a las reglas del progreso y del avance humano. Esta ideología se establece definitivamente en la Modernidad y se conserva hasta hoy en día. Pero esta ideología descuida el aspecto que la Ilustración fue en primer lugar letrada y su representación fue la Enciclopedia: la recopilación del conocimiento para aumentar y difundir el conocimiento humano y así contribuir a su desarrollo: savoir, raison y sensibilité son los tres pilares de la Ilustración francesa y la novela puede reunir en sí una sensibilidad capaz de transmitir al lector el saber y la razón en forma emotiva. Por ello, los filósofos recurren a la novela para transportar los ideales de la Ilustración. Así, Diderot alaba a Richardson, al autor de Pamela, or virtue rewarded (1740) y de Clarissa or the History of a Joung Lady (1748-1751), en su famoso éloge:

O Richardson! ... j'ais, au sortir de la lecture, ce qu'est un homme à la fin d'une journée qu'il a employée à faire le bien.

J'avais parcouru dans l'intervalle de quelques heures un grand nombre de situations, que la vie la plus longue offre à peine dans toute sa durée. J'avais entendu les vrais discours des passions; j'avais vu les ressorts de l'intérêt et de l'amour-propre jouer en cent façons diverses; j'étais devenu spectateur d'une multitude d'incidents, je sentais que j'avais acquis de l'expérience. (Eloge à Richardson, OC, XIII, 193).

Diderot obtiene de la lectura una experiencia fundamental sobre la vida y esta fórmula nos lleva a aquello que caracteriza a la literatura y a las disciplinas en cuestión: ambas se ocupan del individuo y su situación en el mundo. Mientras la literatura en forma intencional o intuitiva, en forma general y particular basándose en la ficción, en un juego de representaciones nos sumerge en un mundo de innumerables posibilidades y experiencias, la teoría tiene el deber y la tarea de sacar a luz los diversos discursos y el vasto y complejo conocimiento de lo inscrito en cada línea o palabra en la literatura. Así, como el físico lee al mundo, y a través de sus teorías, métodos y experimentos formula leyes que rigen al universo, de igual forma las Humanidades y las Ciencias Sociales deben objetivizar y hacer accesible ese conocimiento, muchas veces críptico de la literatura y del arte. Las Humanidades y 
las Ciencias Sociales tienen la función de decodificadores, descifradores del mundo de los signos.

La literatura se transforma en el siglo XVIII, como lo demuestra el elogio de Diderot a la contribución de la novela para la descripción de la vida y del sujeto, como una fuente de conocimiento tan legítima como otro tipo de fuentes. En la literatura, por ejemplo en la novela, encontramos representaciones de sistemas normativos, de valores morales, religiosos, sociales, de sistemas discursivos y de diversos dispositivos, como lo demuestra Foucault en sus obras. La literatura es por ello una transportadora de informaciones vitales de una época. Le Goff (1978/21988, 38) formula en el contexto de la nouvelle histoire:

L'histoire nouvelle a élargi le champ du document historique; à l'histoire de Langlois et de Seignobos essentiellement fondée sur les textes, sur le document écrit, elle a substitué une histoire fondée sur une multiplicité de documents: écrits de toutes sortes, documents figurés, produits de fouilles archéologiques, documents oraux, etc. Une statistique, une courbe des prix, une photographie, un film ou, pour un passé plus lointain, du pollen fossile, un outil, un ex-voto sont, pour l'histoire nouvelle, des documents de premier ordre.

Agrega que para lograr captar el conocimeinto de una época es necesario la transdisciplinariedad y aquí juegan las Humanidades y las Ciencias Sociales un papel fundamental:

Puis c'est le regard chez le voisin, avec l'espoire de faire dialoguer les "frères qui s'ignorent », qui sont presque autant de déceptions, une certaine faillite de la linguistique qui permet pourtant aux historiens de " découvrir un coin du passé sans textes par une induction bornée peut-être mais forte, solide, fondée sur une expérience inattaquable » [...] et puis cet ensemble de domianes repliés sur eux-mêmes par la faute de spécialist sans horizons et sans problèmes, qui maintiennent loin de la faim des historiens de l'historie nouvelle des champs essentiels: la littérature, la philosophie, l'art, les sciences. (Le Goff $1978 /{ }^{21988,44-45)}$

Sus temas y personajes son representaciones de sistemas en debate, en competencia, en conflicto y, por ello, por ejemplo, novelas chilenas como El daño (1997) de Andrea Maturana, Cuando éramos inmortales (1998) de Arturo Fontaine, El revés del alma (2002) y La mujer de mi vida (2005) de Carla Guelfenbein, La ley natural (2004) de Carlos Contreras, Madre que estás en los cielos (2004) de Pablo Simonetti o El desierto (2005) de Carlos Franz representan una precisa radiografía y diagnóstico de la sociedad chilena postdictatorial, y con ello transportan campos tabuizados o limitados en el discurso público y oficialista y un conocimiento fundamental para comprender la sociedad chilena actual.

La cultura en general, la literatura y el arte son campos estratégicos de la Ilustración y del desarrollo y manifestación de un sujeto autónomo, reflexivo y por ello responsable, como Kant lo formula en su famoso lema de 1783: "Aufklärung ist der Ausgang des Menschen aus seiner selbstverschuldeten Unmündigkeit" (1983, 
53) ("Ilustración es la salida del ser humano de su inocencia e inmadurez producida por su propia culpa")

Para terminar: espero que mi exposición haya, aunque brevemente, demostrado no solamente un camino de cómo se podrían reformar las Humanidades y las Ciencias Sociales, sino que haya además puesto en claro la necesidad de las Humanidades, del conocimiento que éstas conllevan y al fin la necesidad de la transciplinariedad.

Por ello, debo subrayar que

una universidad sin Humanidades es como un cuerpo sin pulmón.

Las Humanidades son imprescindibles para la institución universidad y para el desarrollo de una sociedad civil y democrática, por lo tanto se deben mantener su autonomía y legitimación, para esto deben, eso sí, reformarse y someterse a serios criterios de excelencia.

Para terminar indico que lo expuesto nace de la realidad actual que exige este tipo de medidas, pero que a la vez tiene su origen en mi más profunda admiración por Chile, en mi entrañable afecto por el país, que tiene fuerza, visiones y el deseo de enfrentar todos estos problemas aludidos.

Mientras Chile ha solucionado un problema básico como aquel de la macroeconomía, debe pues ahora solucionar el problema de la microeconomía, el de la pobreza, del aún significativo analfabetismo, y debe reformar la educación desde su base a su tope. Una tarea nada fácil, pero si Chile sabe invertir las riquezas que da la globalización a raíz de y los altos precios que se pagan por la energía y la materia prima, entonces Chile lo logrará. Por ello propongo el lema "Chile está" (está en el mundo dinámico y surgente) y les deseo paciencia, perseverancia, sabiduría y mucha suerte en este escabroso camino.

\section{BIBLIOGRAFÍA}

\section{Autores}

Borges, Jorge Luis. (1925). Inquisiciones. Buenos Aires.

—. (1926). El tamaño de mi esperanza. Buenos Aires.

—. (1928). El idioma de los argentinos. Buenos Aires.

——. (1972/21989). Obras Completas (=OC). Buenos Aires.

Diderot. (1951). “Eloge à Richarson”, en: id. Euvre. Paris. pp. 1060-1074.

Contreras, Gonzalo. (2004). La ley natural. Santiago de Chile.

Fontaine, Arturo. (1998). Cuando éramos inmortales. Santiago de Chile. 
Franz, Carlos. (2005). El desierto. Barcelona.

Guelfenbein, Carla. (2002/2005). El revés del alma. Chile.

Guelfenbein, Carla. (2005). La mujer de mi vida. Santiago de Chile.

Maturana, Andrea. (1997). El daño. Santiago de Chile.

Simonetti, Pablo. (2004). Madre que estás en los cielos. Santiago de Chile.

II. Estudios

Baudrillard, Jean. (1981). Simulacres et simulation. Paris.

Bhabha, Homi K. (1994). The Location of Culture. London/New York.

Brockhaus Multimedial. (2005).

Czarnecki, Lukas. http:/ /www.hpwt.de/Quanten2.htm. (2003)

Deleuze, Gilles/Guattari, Felix. (1976). Rhizome. Paris.

Deutsch, David. (1996). Die Physik der Welterkenntnis. Auf dem Weg zum universellen Verstehen. Basel/Boston/Berlin.

DeWitt, Brice Seligman/Graham, Neill. (eds.). (1973). The Many-Worlds Interpretation of Quantum Mechanics. A Fundamental Exposition by Hug Everett, III, with Papers by J.A. Wheeler, B.S. deWitt, L.N. Cooper and D. van Vechten and N. Graham. Princeton Series Physics. Princeton/New Jersey.

Everett III, Hugh. (s.f.). Hugh Everett III and the Many Worlds Theory. The Macrocosm of Many Worlds, en: http:/ / everythingforever.com/everett.htm.

Fichte, Johann Gottlieb. (1807/ 1960). “Deduzierter Plan einer zu Berlin zu errichtenden höheren Lerhanstalt, die in gehöriger Verbindung mit einer Akademie der Wissenschaften stehe“, en: Weischedel, Wilhlen. (1969). Idee und Wirklichkeit einer Universität. Dokumente zur Geschichte der Friedrich-Wilhelm-Universität zu Berlin. Berlin. pp. 0-105; vid. también Müller (1990).

Frühwald, Wolfgang/Jauß, Hans Robert/Koselleck, Reinhart/Mittelstraß, Jürgen/ Steinwachs, Burkhart. (1991/21996). Geisteswissenschaften heute. Eine 
Denkschrift. Frankfurt am Main.

Foucault, Michel. (1966). Les mots et les choses. Paris.

García Canclini, Néstor. (1990/1992/21995). Culturas híbridas. Estrategias para entrar y salir de la modernidad. México Buenos Aires.

García Canclini, Néstor. (1996). "Cultural Studies Questionnaire", en: Journal of Latin American Cultural Studies, vol. 5, No. 1: 83-87.

Gayer, Paul. (1997). "Für eine Kritische Theorie der Literaturwissenschaft", en: Hunfeld, Hans. (ed.). Wozu Wissenschaft heute? Tübingen. pp. 71-90.

Le Goff, Jacques. (1978/21988). La nouvelle histoire. Bruxelles.

Hall, Stuart. (1990). "The Emergence of Cultural Studies and the Crisis of the Humanities", en: October, 53: 11-23.

Habermas, Jürgen. (1981). Theorie des kommunikativen Handelns. Handlungsrationalität und gesellschaftliche Rationalisierung / Zur Kritik der funktionalistischen Vernunft. Bd. I/II. Frankfürt am Main.

Johnson, Richard. (1986/87). “What is Cultural Studies Anyway?", en: Social Text. 16, (winter): 38-80.

Kant, Immanuel. (1783/1983). “Beantwortung der Frage: Was ist Aufklärung?”, en: Werke. Schriften zur Anthroplogie, Geschichtsphilosophie, Politik und Pädagogik. Erster Teil. Vol 9. Darmstadt. pp. 53-61

Kant, Immanuel. (1798/1983a). “Der Streit der Fakultäten”, en: Werke. Schriften zur Anthroplogie, Geschichtsphilosophie, Politik und Pädagogik. Erster Teil. Vol. 9. Darmstadt. pp. 262-393.

Lyotard, Jean-François. (1979). La condition postmoderne. Rapport sur le savoir. Paris.

Manovich, Lev. (2003). “New Media from Borges to html”, en: Noa Wardrip-Fruin/ Nick Montfort. (eds.). The New Media Reader. Cambridge/Massachusetts/ London. pp. 13-25.

Mittelstraß, Jürgen. (1978). "Sozialwissenschaft im System der Wissenschaft", en: M. Timmernann. (ed.). Sozialwissenschaften. Eine multidisziplinäre 
Einführung. Konstanz. pp. 173-189.

Mittelstraß, Jürgen. (1986). “Wissenschaft als Kultur", en: Heidelberger Jahrbücher. 39: 51-71.

Mittelstraß, Jürgen. (1989). Glanz und Elend der Geisteswissenschaften. Oldenburg.

Müller, Ernst. (ed.). (1990). Gelegentliche Gedanken über Universitäten von J. J. Engel, J. B. Erhard, F. A. Wolf, J. G. Fichte, F. D. E. Schleiermacher, K. F. Savigny, W. v. Humboldt, G. F. W. Hegel. Leipzig.

Murray, Janet H. (2003). "Inventing the Medium", en: Noa Wardrip-Fruin/Nick Montfort. (eds.). The New Media Reader. Cambridge/Massachusetts/ London. pp. 7-11.

Nelson, Theodor H. (1965/2003). "A File Structure for the Complex, the Changing, and the Indeterminate", en: Noa Wardrip-Fruin/Nick Montfort. (eds.). The New Media Reader. Cambridge/Massachusetts/London. pp. 134-145.

Nelson, Theodor H. (1974/2003). "Computer Lib/Dream Machines", en: Noa Wardrip-Fruin/Nick Montfort. (eds.). The New Media Reader. Cambridge/ Massachusetts/London. pp. 303-338.

Nelson, Theodor H. (1981/2003). “From Literary Machines. Proposal for a Universal Electronic. Publishing System and Archive", en: Noa Wardrip-Fruin/Nick Montfort. (eds.). The New Media Reader. Cambridge/Massachusetts/ London. pp. 443-461.

Noa Wardrip-Fruin/Nick Montfort. (eds.). The New Media Reader. Cambridge/ Massachusetts/ London.

Toro, Alfonso de. (2003). "Jenseits von Postmoderne und Postkolonialität. Materialien zu einem Modell der Hybridität und des Körpers als transrelationalem, transversalem und transmedialem Wissenschaftskonzept", en: Christoph Hamann/Cornelia Sieber (eds.). Räume der Hybridität. Zur Aktualität postkolonialer Konzepte. Hildesheim/ Zürich/ New York. pp. 15-52.

Toro, Alfonso de. (2004). "Zu einer Kulturtheorie der Hybridität als transrelationales, transversales und transmediales Wissenschaftssystem", en: Iberoromania 59: $1-42$.

Toro, Alfonso de. (2004a). “Hacia una teoría de la cultura de la hibridez como sistema 
científico transrelacional, 'transversal' y 'transmedial'", en: Estudios Literarios \& Estudios Culturales. Nuevo Texto Crítico (Stanford University) 25/26: 275-329.

Toro, Alfonso de. (2006). “Borgesvirtual. El creador de los medios virtaules-digitales y de la teoría de Diversos mundos Escritura - percepción - compresión Implosión - expansión la enciclopedia navegante o The Navigation of the user in the web", en: Aisthesis. Revista Chilena de Investigaciones Estéticas. Julio, Nr. 39: 49-71.

Toro, Alfonso de. (2007). “Borgesvirtuell. Der Schöpfer digital-virtueller Medien und der Vielen-Welten-Theorie. Schrift - Wahrnehmung - Verdichtung - Implosion - Ausdehnung. Die navigierende Enzyklopädie oder The Navigation of the User in the Web", en: Felten, Uta/Isabel Maurer Queipo (Hrsg.): Intermedialität in Hispanoamerika: Brüche und Zwischenräume. Tübingen: Stauffenburg. pp. 221-242.

Schleiermacher, F. D. (1808/1960). “Gelegentliche Gedanken über Universitäten in deutschem Sinn. Nebst einem Anhang über eine neue zu errichtende", en: Weischedel, Wilhelm. (ed.). (1969). Idee und Wirklichkeit einer Universität. Dokumente zur Geschichte der Friedrich-Wilhelm-Universität zu Berlin. Berlin. pp. 106-192; vid. también Müller (1990).

Vidal, Hernán. (1996). “Los derechos humanos, hermenéutica para la crítica literaria y los estudios culturales latinoamericanos: informe de una experiencia”, en: Revista Iberoamericana. Vol. LXII (Julio-Diciembre). No. 176-177: 719-729.

Weischedel, Wilhelm. (1969). Idee und Wirklichkeit einer Universität. Dokumente zur Geschichte der Friedrich-Wilhelm-Universität zu Berlin. Berlin. pp. 106192; vid. también Müller (1990).

Weizenbaum, Joseph. (1976/2003). "From Computer Power and Human Reason. From Judgment to Calculation", en: Noa Wardrip-Fruin/Nick Montfort. (eds.). The New Media Reader. Cambridge/Massachusetts/London. pp. 367-375. 